\author{
Luigi Bisio* - Piero GiUnTELLI**
}

\title{
I Carabidi della Val Corsaglia, della Val Casotto e della Valle Mongia (Alpi Liguri) (Coleoptera Carabidae)
}

\begin{abstract}
Riassunto: Dopo avere brevemente illustrato i principali caratteri geologici, climatici e vegetazionali della Val Corsaglia, della Val Casotto e della Valle Mongia, gli autori propongono una sintesi delle ricerche carabidologiche condotte sino a oggi in quest'area. Viene presentato un catalogo topografico delle 159 specie di Carabidi (Cicindelinae incluse) segnalate in questo territorio, con note riguardanti l'ecologia e la corologia di quelle più interessanti. Infine, sono descritte le principali carabidocenosi osservate.
\end{abstract}

\begin{abstract}
Carabid beetles of the Corsaglia, Casotto and Mongia Valleys (Ligurian Alps, Piedmont, Cuneo, Italy) (Coleoptera Carabidae). After a short illustration of the main geological as well as climatic and vegetational features of the Corsaglia, Casotto and Mongia Valleys, a synthesis of the carabidological researches carried out so far in this area is reviewed. A topographic catalogue of the 159 Carabid species (Cicindelinae included) recorded from this territory is given, with notes regarding the ecology and the distribution of the most interesting ones. Furthermore, the main observed carabid assemblages are described.
\end{abstract}

Key words: Coleoptera Carabidae, Alpine fauna, Western Alps, Piedmont, Cuneo, Corsaglia, Casotto and Mongia Valleys.

\section{INTRODUZIONE}

Dopo i precedenti contributi sulla carabidofauna di alcune valli delle Alpi Liguri (Bisio et al., 2013, 2015b, 2018; Bisio \& Giuntelli, 2018), gli autori intendevano dedicare questa nota ai Carabidi (Cicindelinae incluse) della Val Corsaglia e della Val Casotto, valli nelle quali gli scriventi hanno condotto, nel corso di circa due anni, una serie di ricerche volte a incrementare le conoscenze sulla carabidofauna ivi presente oltre quelle già citate in letteratura. Dopo il recente rinvenimento di Duvalius chestai Casale, Giachino, Lana, 2019 nella vicina Valle Mongia (cfr. Casale et al., 2019), situata a Est della Val Casotto, gli autori hanno ritenuto opportuno inserire anche questa valle nel presente lavoro.

Sebbene queste valli siano state meno frequentate dagli entomologi rispetto ad altre delle Alpi Liguri quali la Val Pesio e la Val Tanaro, i dati disponibili riguardanti il loro territorio sono comunque relativamente numerosi. I primi contributi risalgono alla seconda metà del XIX secolo e furono merito di Baudi di Selve (1871, 1890), K. Daniel \& J. Daniel (1898) e Ganglbauer (1900). Questo primo nucleo di dati venne incrementato nel corso della prima metà del secolo scorso, talora anche solo con singole segnalazioni, da
K. Daniel (1906), Dellepiane (1924), Luigioni, (1929), Müller (1930), Schatzmayr (1930), Breuning (19321936), Binaghi (1936, 1939) e Capra (1941). Un discreto numero di taxa rinvenuti in stazioni del territorio in oggetto è stato poi segnalato da Magistretti (1965) nel suo ormai classico catalogo topografico sinonimico.

Dopo il contributo di quest'ultimo autore, si sono avute altre segnalazioni ad opera di Benazzi \& Gourbault (1977), Bisio (2000, 2002 2007, 2008, 2009b, 2009c), Bologna \& Vigna Taglianti (1982, 1985), Bordoni (1968), Casale \& Cavazzuti (1975, 1976), Casale \& Vigna Taglianti (1993), Giachino (1993), Giachino \& Casale (1983), Hieke (1978), Martinotti (1968), Morisi (1969, 1970, 1971a, 1971b; 1972a, 1972b, 1973a, 1973b), Peano (1973, 1974), Sciaky (1984), Vigna Taglianti (1966), Vigna Taglianti \& Casale (1973) e Vigna Taglianti et al. (2001).

A completare il quadro delle conoscenze faunistiche sulle specie di Carabidi presenti in questo territorio hanno poi contribuito sia la recente CKmap (Casale et al., 2006), sia i successivi lavori di Casale \& Giachino (2010), Lana et al. (2014), Bisio et al. (2017) e Casale et al. (2019).

Questa nota intende presentare una sintesi dei

*Luigi Bisio, Via Galilei 4, 10082 Cuorgnè (TO), Italia. E-mail: luigibisio@virgilio.it

**Piero Giuntelli, Via Torino 160, 10076 Nole Canavese (TO), Italia. E-mail: pierogiuntelli@virgilio.it 
risultati delle ricerche carabidologiche condotte sino a oggi in queste valli.

\section{DESCRIZIONE DEL TERRITORIO}

Per Val Corsaglia, Val Casotto e Valle Mongia qui si intendono i bacini idrografici dei torrenti omonimi, considerati rispettivamente dalle sorgenti alla confluenza dei primi due nei pressi di San Michele Mondovì e dalle sorgenti del Torrente Mongia alla sua confluenza nel Corsaglia poco a valle dello stesso abitato, limiti oltre i quali il Corsaglia (che nell'accezione comune è il torrente principale) scorre ormai in un paesaggio collinare. Le tre valli (Figg. 1-3) si sviluppano parallele da Sud verso Nord a ridosso del versante settentrionale dello spartiacque che divide la Val Tanaro dal territorio monregalese (la cresta Monte
Mongioie-Bocchin d'Aseo-Monte Rotondo-Bric Conoia-Pizzo d'Ormea-Colle dei Termini-Cima Ciuaiera-Monte Antoroto-Monte Berlino-Colla di Casotto-Bric Mindino-Bric del Prato rotondo-Bric Ciarandella). Il tratto intermedio della dorsale (tra il Mongioie e il Bric Mindino) forma l'ossatura della testata delle tre valli, mentre quello terminale (oltre il Bric Mindino) delimita invece a Est il versante destro della Val Mongia e la separa dalla Val Tanaro. Il punto più elevato delle valli è la vetta del Monte Mongioie (2630 m), mentre l'abitato di San Michele Mondovì, situato a $444 \mathrm{~m}$ di quota nei pressi dell'imbocco delle stesse, è quello più basso.

Dall'esame della Carta geologica d'Italia 1:100.000 (Fogli n 81 Ceva, $n^{\circ} 91$ Boves e n $92 \mathrm{Al}-$ benga) e dalla letteratura più recente (Dal Piaz, 1991;

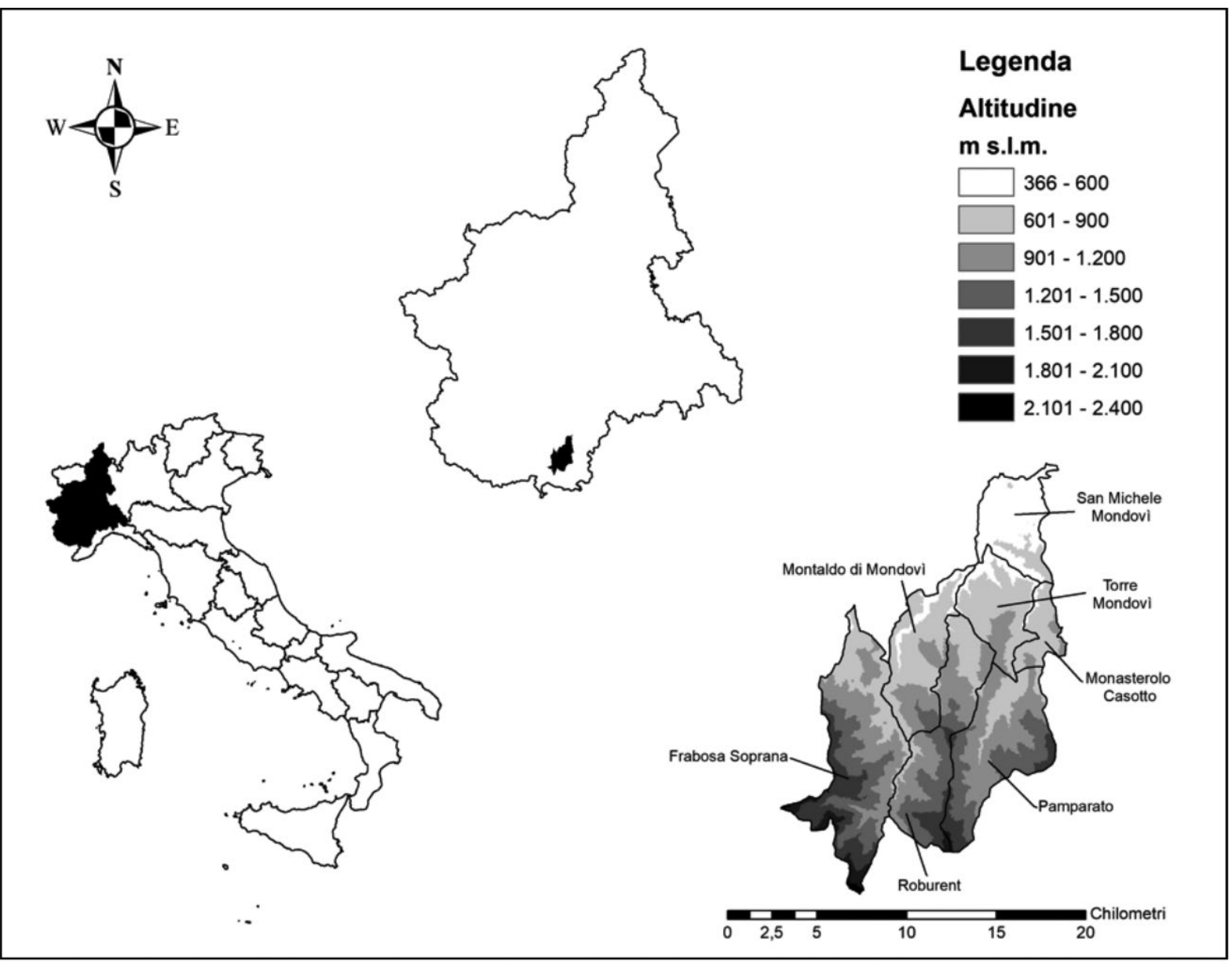

Fig. 1. La Val Corsaglia e la Val Casotto, territorio oggetto della ricerca (tavola redatta da Matteo Negro). 
Peano, 1999) si può desumere che il territorio in oggetto è inciso nelle falde appartenenti alla Zona Brianzonese (Pennidico). Le litologie più diffuse sono le seguenti:

1. Porfiroidi quarziferi del tegumento permo-carbonifero: rappresentano il litotipo prevalente e affiorano in tre grandi formazioni rispettivamente in corrispondenza della testata della Val Corsaglia (massiccio del Pizzo d'Ormea e suoi contrafforti), lungo un'ampia fascia che dalla testata e dalla destra orografica della Val Casotto attraversa diagonalmente le due valli da Est-Sudest a Ovest-Nordovest (dalla cresta Monte Berlino-Colla di Casotto-Bric MindinoBric Neirassa all'abitato di Valcasotto, al Monte Alpet e all'abitato di Corsaglia) e nella bassa Val Casotto (a valle di Pamparato);

2. Formazioni calcaree, calcareo-dolomitiche, calcareo-marnose delle coperture meso-cenozoiche: di minore estensione, interrompono la continuità dei porfiroidi rispettivamente lungo i contrafforti del Massiccio del Mongioie, in una fascia che attraversa le tre valli in direzione Est-Sudest/Ovest-Nordovest (dal Monte Antoroto all'abitato di Bossea e al Monte Mondolè), nella bassa Val Corsaglia (nei dintorni di Roburent) e in Valle Mongia;

3. Calcescisti della Zona Piemontese: affiorano in corrispondenza della bassa Val Corsaglia (nei dintorni di Montaldo Mondovì);

4. Marne e Arenarie del complesso pelitico-arenaceo miocenico: sono presenti nei pressi della

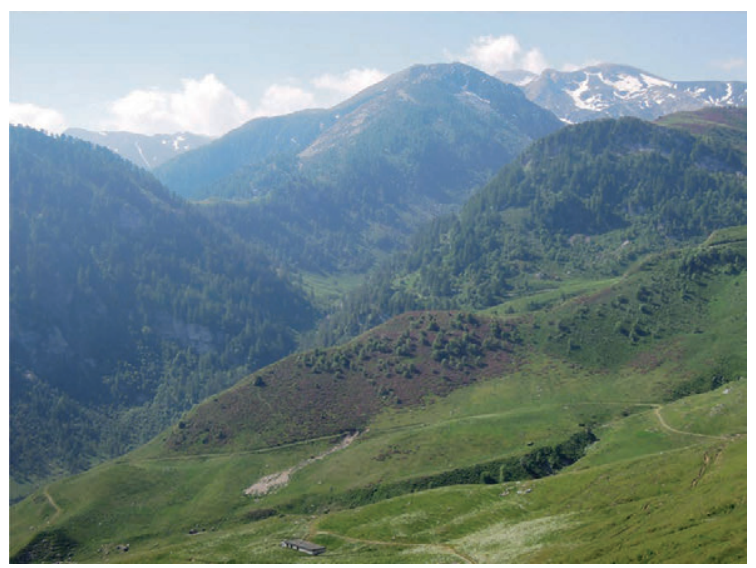

Fig. 2. L'alta Val Corsaglia vista dal Colle della Balma (foto Luigi Bisio 13.VI.2017). confluenza delle Valli Corsaglia e Casotto e nei dintorni di San Michele Mondovì.

L'eterogeneità delle rocce affioranti nelle diverse zone del territorio in oggetto determina evidenti contrasti morfologici. Alla testata delle tre valli, soprattutto in Val Corsaglia, la resistenza all'alterazione dei porfiroidi caratterizza la morfologia delle creste modellate in questo litotipo, che sono rocciose e culminanti nella piramide del Pizzo d'Ormea. In questa parte di territorio vallivo sono anche ben conservate le morfologie di esarazione e di deposito conferito dal glacialismo pleistocenico, rappresentate da diversi circhi glaciali (Fig. 4). Dove affiorano invece le forma-

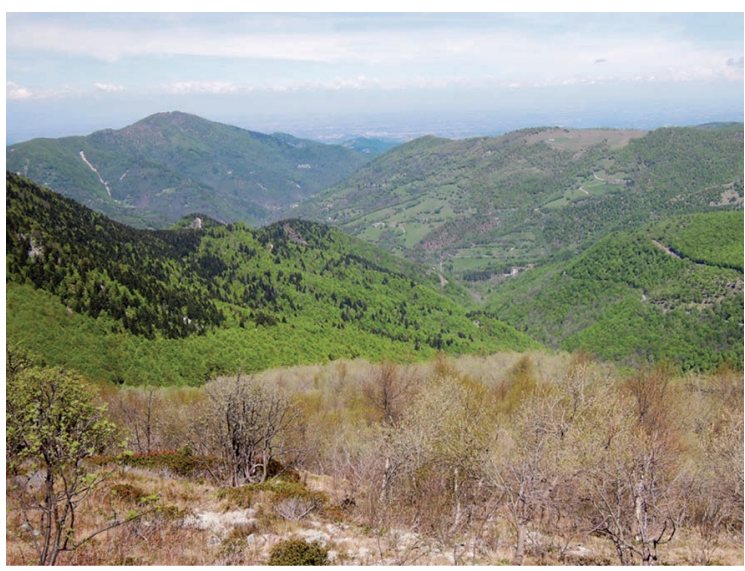

Fig. 3. La Val Casotto vista dal Monte Berlino (foto Luigi Bisio 15.V.2017).

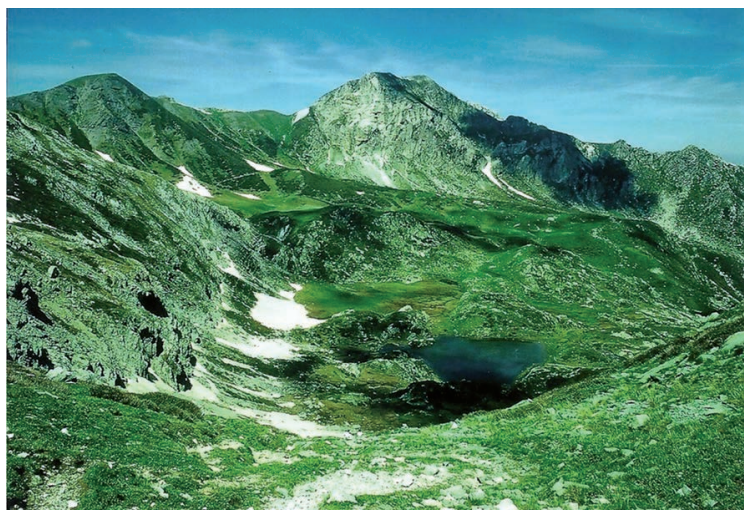

Fig. 4. Il circo glaciale dei Laghi della Brignola, visto dal colle omonimo (foto Luigi Bisio 30.VI.1991). 
zioni carbonatiche il concorso dei fattori topografici (la quota e l'esposizione settentrionale) e climatici (l'abbondanza delle precipitazioni) hanno favorito $i$ processi di dissoluzione dando origine a un sistema carsico di fratture e di cavità sotterranee delle quali fa parte la ben nota Grotta di Bossea (a tale proposito cfr. Gregoretti, 1991; Peano, 1999).

Per il territorio in oggetto sono disponibili dati pluviometrici (Regione Piemonte, 1998) relativi alle stazioni di Torre Mondovì, Montaldo Mondovì, Prà di Roburent, San Giacomo di Roburent e Pamparato. Il quadro riassuntivo della Tab. 1, ottenuta dall'elaborazione di tali dati, evidenzia differenze importanti al variare della quota. Nella stazione di Torre Mondovì (a $470 \mathrm{~m}$ di quota, in corrispondenza dell'imbocco della Val Corsaglia) il clima risulta tendenzialmente più xerico, caratterizzato da un tasso medio annuo di precipitazioni che non raggiunge i $1000 \mathrm{~m}$; risalendo le valli, invece, il clima diventa più umido e le quantità medie annue di precipitazioni tendono ad aumentare sensibilmente sino a livelli compresi tra i 1300-1400 $\mathrm{mm}$ nelle due stazioni nei dintorni di Roburent (poco al di sopra dei $1000 \mathrm{~m}$ di quota). Pur non essendo disponibili misure nel settore della testata, è plausibile ritenere che, più a monte di queste ultime stazioni, si raggiungano medie annuali superiori.

Il regime pluviometrico delle tre valli (Fig. 5) è caratterizzato da un massimo assoluto autunnale nel

Tab. 1. Dati pluviometrici relativi al territorio in oggetto.

\begin{tabular}{lcccc}
\hline Stazione & Quota & Periodo & Precipitazioni & Precipitazioni \\
\hline & & & medie annue, mm & medie trimestre estivo, mm \\
\hline Torre Mondovì & $470 \mathrm{~m}$ & $1927-1981$ & 990 & 184 \\
Pamparato & $782 \mathrm{~m}$ & $1915-1979$ & 1151 & 203 \\
Montaldo Mondovì & $796 \mathrm{~m}$ & $1924-1941$ & 1202 & 210 \\
S. Giacomo di Roburent & $1011 \mathrm{~m}$ & $1940-1964$ & 1315 & 219 \\
Prà di Roburent & $1014 \mathrm{~m}$ & $1930-1973$ & 1472 & 275 \\
\hline
\end{tabular}

Val Corsaglia e Val Casotto: medie mensili delle precipitazioni

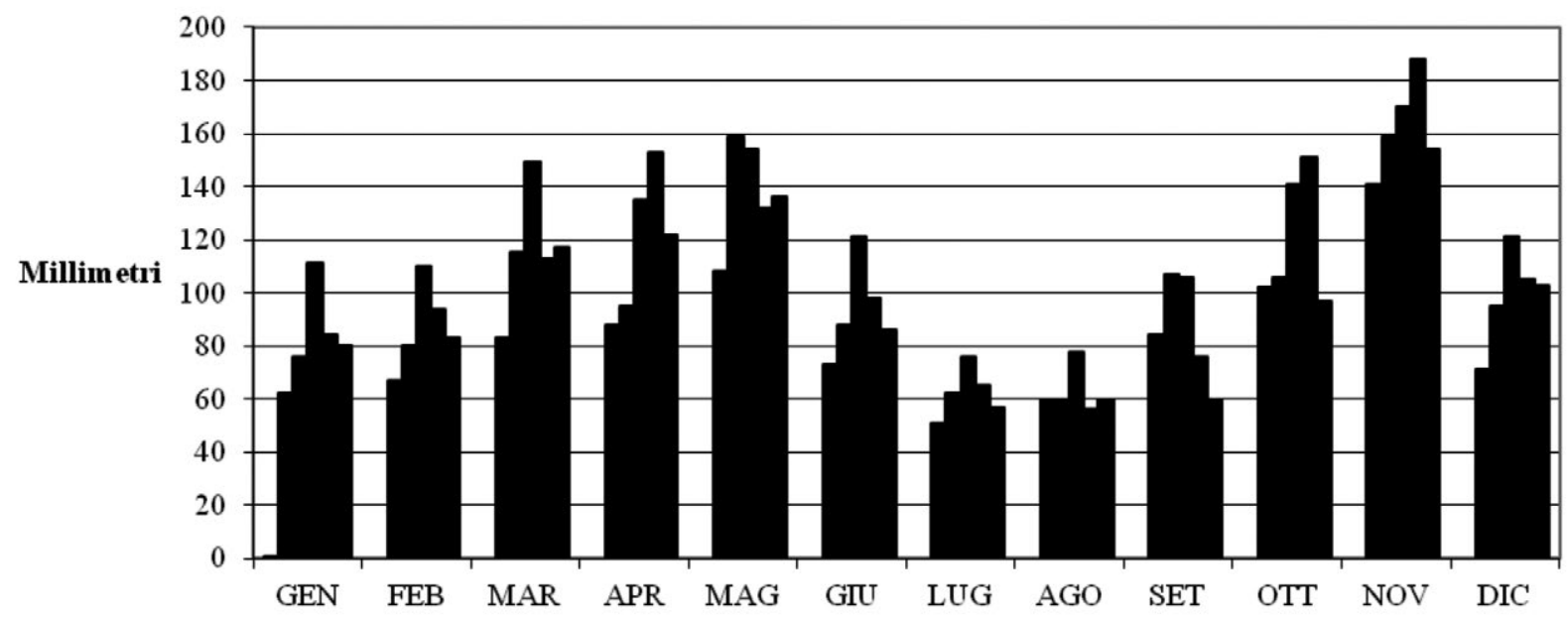

Fig. 5. Regime pluviometrico del territorio in oggetto. 
mese di novembre in tutte le stazioni e un massimo relativo primaverile (nel mese di maggio a Torre Mondovì, Pamparato, Montaldo Mondovì e Prà di Roburent, nel mese di aprile a San Giacomo di Roburent). Il minimo assoluto è estivo (nel mese di luglio o agosto nelle diverse stazioni, con differenze comunque del tutto trascurabili tra i due mesi), mentre quello relativo è invernale (a gennaio a Torre Mondovì, Pamparato, Prà di Roburent e San Giacomo di Roburent, a febbraio a Montaldo Mondovi).

Il trimestre estivo (giugno-luglio-agosto) risulta caratterizzato da valori di piovosità complessivi tendenzialmente bassi, $184 \mathrm{~mm}$ nella stazione di Torre Mondovì, tra 203 e 275 nelle altre stazioni. Tali valori fanno pensare a problemi di deficit idrico estivo (soprattutto nei mesi di luglio e agosto, i più critici) che sono certamente più marcati verso l'imbocco delle valli. Nonostante gli apporti meteorici estivi non molto elevati, la situazione pare invece decisamente diversa verso la parte alta delle valli. L'elevata quantità di precipitazioni (tra 398 e $438 \mathrm{~mm}$ ) registrate nei dintorni di Roburent nel corso del trimestre primaverile (marzo-aprile-maggio) porta infatti a ritenere che, più a monte di quest'ultima località, si raggiungano medie trimestrali superiori e che, più in quota, gli apporti meteorici della primavera siano in gran parte nevosi. 亡̀ probabile che la graduale fusione (che lungo i pendii delle testate delle tre valli è certamente rallentata dall'esposizione settentrionale) delle masse nevose accumulate in tale periodo contribuisca a sopperire in modo sensibile alla scarsità di precipitazioni nel periodo estivo.

La copertura forestale e arbustiva che caratterizza le tre valli è la diretta conseguenza delle caratteristiche climatiche. A partire dall'imbocco si osserva la seguente successione vegetazionale:

1. un orizzonte submontano (che si estende lungo i fondivalle dagli imbocchi delle tre valli sino a Pamparato e a Bossea e che risale gli opposti versanti) occupato da boschi di castagno $(\mathrm{Ca}$ stanea sativa), da boschi misti di latifoglie e da formazioni aperte rappresentate soprattutto da prati stabili da sfalcio;

2. un orizzonte montano inferiore rappresentato da boschi misti di faggio (Fagus sylvatica) e abete bianco (Abies alba) che si alternano a prati e pascoli e a consorzi misti di latifoglie;

3. un orizzonte subalpino occupato soprattutto da ontaneti ad Alnus viridis;
4. un orizzonte alpino contraddistinto da un'ampia estensione della prateria alpina la cui continuità è di fatto interrotta in modo significativo solo in corrispondenza degli affioramenti rocciosi che caratterizzano la dorsale della sinistra orografica.

\section{Catalogo Topografico}

Per la nomenclatura di quasi tutte le specie e per l'attribuzione del corotipo di competenza a ciascuna di esse, si è fatto riferimento alla Checklist dei Carabidi italiani elaborata da Vigna Taglianti (2005), apportando peraltro alcune variazioni che sono di volta in volta giustificate con nota a pié di pagina. Invece, per quanto riguarda la nomenclatura dei Bembidiini, si è assunta come base la Checklist nel contributo più recente di Neri et al. (2011). Per ogni località segnalata viene indicata la citazione bibliografica o il raccoglitore (GA=Gianni Allegro; $\mathrm{LB}=$ Luigi Bisio; $P G=$ Piero Giuntelli). Si precisa che con "Val Casotto" si intende l'intera valle, mentre con "Valcasotto" si intende solo l'abitato con questo nome.

1. Aptinus (Aptinus) alpinus Dejean \& Boisduval, 1829

Val Casotto: Colla di Casotto m 1450 (LB); Monte Berlino m 1500-1700 (LB).

COROTIPO: SW-Alpino (ALSW).

2. Brachinus (Brachinus) elegans Chaudoir, 1842 Val Corsaglia: Moline (Torre Mondovì) m 500 (LB). CoRotipo: Mediterraneo (MED).

3. Brachinus (Brachynidius) explodens Duftschmid, 1812

Val Corsaglia: Moline (Torre Mondovì) m 500 (LB). Val Casotto: Tetti Casotto (San Michele Mondovì) m 500 (LB).

Corotipo: Asiatico-Europeo (ASE).

4. Brachinus (Brachynidius) glabratus Latreille \& Dejean, 1822

Val Casotto: Tetti Casotto (San Michele Mondovì) $\mathrm{m}$ 500 (LB); Pamparato m 900 (LB).

Corotipo: S-Europeo (SEU).

5. Brachinus (Brachynidius) sclopeta (Fabricius, 1792) 
Val Corsaglia: Moline (Torre Mondovì) m 500 (LB). COROTIPO: Europeo-Mediterraneo (EUM).

6. Cicindela (Cicindela) campestris campestris Linné, 1758

Val Corsaglia: Monte Mondolè (Magistretti, 1965).

Val Casotto: Monte Berlino m 1500-1700 (LB). Corotipo: Paleartico (PAL).

7. Cicindela (Cicindela) gallica Brullé, 1834

Val Corsaglia: Pizzo d'Ormea (Magistretti, 1965); Laghi della Brignola m 2100 (LB \& PG); Monte Mondolè (Magistretti, 1965).

Corotipo: Centroeuropeo (CEU).

8. Carabus (Carabus) granulatus interstitialis Duftschmid, 1812

Val Corsaglia: San Giacomo di Roburent m 1000 (Vigna Taglianti et al., 2001; Casale et al., 2006); Corsaglia $\mathrm{m} 700$ (LB).

Val Casotto: Colla di Casotto m 1450 (LB).

COROTIPO: Asiatico-Europeo (ASE).

9. Carabus (Archicarabus) monticola Dejean, 1826

Val Corsaglia: Monte Mondolè (Casale et al., 1982).

Val Casotto: Colla di Valcasotto m1400-1600 (Casale et al., 2006).

COROTIPO: W-Alpino (ALPW).

10. Carabus (Mesocarabus) problematicus inflatus Kraatz, 1878

Val Corsaglia: Pizzo d'Ormea (Breuning 1932-1936: sub problematicus dellabeffae; Casale et al., 2006); Monte Mondolè $\mathrm{m} 1900$ (LB \& PG).

Val Casotto: Colla di Casotto (Casale et al., 2006). COROTIPO: Europeo (EUR).

11. Carabus (Orinocarabus) pedemontanus pedemontanus Ganglbauer, 1891

Val Corsaglia: Laghi e Bocchetta della Brignola m 2100-2300 (Bisio, 2000); Laghi della Brignola m 2150 (LB \& GA); Monte Mondolè (Magistretti, 1965: sub

La specie è stata inclusa da Deuve (2004) nel subg. Pachystus. putzeysianus pedemontanus); Monte Mondolè m 1900-2300 (Casale \& Cavazzuti, 1976: sub putzeysianus pedemontanus); Monte Mondolè $\mathrm{m} 1900$ (Bisio, 2000: sub putzeysianus pedemontanus).

Val Casotto: Monte Mussiglione m 1950 (LB \& PG). COROTIPO: SW-Alpino (ALSW).

12. Carabus (Oreocarabus ${ }^{1}$ ) glabratus latior Born, 1895

Val Corsaglia: Corsaglia (Casale et al., 2006); San Giacomo di Roburent m 1000 (Casale et al., 2006).

Val Casotto: Valcasotto (Casale et al., 2006); Pamparato $\mathrm{m} 1045$ (Casale et al., 2006); $114 \mathrm{Pi} / \mathrm{CN}$ Tana del Forno (pozzo o grotta dell'Orso) (Pamparato, frazione Serra) m 1045 (Vigna Taglianti, 1966; Morisi, 1972a; Bologna \& Vigna Taglianti, 1985).

COROTIPO: Europeo (EUR).

13. Carabus (Tomocarabus) convexus convexus Fabricius, 1775

Val Casotto: Colla di Valcasotto (Casale et al., 2006). CoROtIPO: Sibirico-Europeo (SIE).

14. Carabus (Chaetocarabus) intricatus Linné, 1761

Val Corsaglia: Moline (Torre Mondovì) m 500 (LB); Corsaglia (Casale et al., 2006); San Rocco (Bossea) m 750 (LB).

COROTIPO: Europeo (EUR).

15. Carabus (Platycarabus) depressus lucens Schaum, 1857

Val Corsaglia: Alta Val Corsaglia (Casale et al., 2006); Pizzo d'Ormea (Casale et al., 2006); Monte Mondolè $\mathrm{m} 1900$ (LB \& PG).

Val Casotto: Monte Antoroto e Monte Berlino (Casale et al., 1982).

Corotipo: Centroeuropeo (CEU).

16. Carabus (Megodontus) germarii fiorii Born, 1901

Val Corsaglia: San Giacomo di Roburent m 1000 (Casale et al., 2006).

Val Casotto: $114 \mathrm{Pi} / \mathrm{CN}$ Tana del Forno (pozzo o grotta dell'Orso) (Pamparato, frazione Serra) m 1045 (Morisi, 1972a: sub Megodontus germari pedemontanensis; Bologna \& Vigna Taglianti, 1985); Colla di Casotto (Casale et al., 2006).

Corotipo: S-Europeo (SEU). 
17. Carabus (Chrysocarabus) solieri liguranus Breuning, 1933

Val Corsaglia: Monte Mondolè $m$ 1800-2000 (Casale et al., 2006); Pizzo d'Ormea m 2400 (Casale \& Cavazzuti, 1975); Pizzo d'Ormea m 2000 (Casale et al., 2006).

Val Casotto: Valcasotto m 950 (LB); Colla di Casotto (Casale \& Cavazzuti, 1975; Casale et al., 2006); Monte Berlino m 1500-1700 (LB \& PG).

Corotipo: SW-Alpino (ALSW).

18. Cychrus italicus Bonelli, 1810

Val Corsaglia: San Giacomo di Roburent m 1000 (Casale et al., 2006); 112 Pi/CN Tana di San Luigi (= Grotta dello Spelerpes) (Roburent) m 780 (Morisi, 1970; Benazzi \& Gourbault, 1977; Bologna \& Vigna Taglianti, 1985).

Val Casotto: $114 \mathrm{Pi} / \mathrm{CN}$ Tana del Forno (pozzo o grotta dell'Orso) (Pamparato, frazione Serra) m 1045 (Morisi, 1972b; Bologna \& Vigna Taglianti, 1985); Monte Berlino m 1500-1700 (LB \& PG).

COROTIPO: Alpino-Appenninico (ALAP).

19. Cychrus attenuatus attenuatus (Fabricius, 1792)

Val Casotto: Colla di Valcasotto (Casale et al., 2006). COROTIPO: Centroeuropeo (CEU).

20. Cychrus angulicollis Sella, 1874

Val Corsaglia: Bocchin d'Aseo (Schatzmayr, 1943); Laghi della Brignola m 2200 (un paio di elitre) (LB \& PG); Monte Mondolè (Magistretti, 1965).

Stenoendemita delle Alpi Cozie meridionali, delle Marittime e delle Liguri, $C$. angulicollis è diffuso prevalentemente nei massicci calcarei dove popola le conche fresche e nevose (Casale et al., 1982).

COROTIPO: SW-Alpino (ALSW).

21. Leistus (Leistus) nitidus (Duftschmid, 1812) Val Casotto: Val Casotto m 1300 (Bisio et al., 2017). Corotipo: Centroeuropeo (CEU).

22. Leistus (Pogonophorus) spinibarbis spinibarbis (Fabricius, 1775)

Val Casotto: Ponte Tetti Casotto (San Michele Mondovì) $\mathrm{m} 500$ (Bisio et al., 2017).

COROTIPO: Europeo (EUR).

23. Nebria (Nebria) brevicollis (Fabricius, 1792)
Val Corsaglia: Corsaglia m 700 (LB); $112 \mathrm{Pi} / \mathrm{CN}$ Tana di San Luigi (= Grotta dello Spelerpes) (Roburent) m 780 (Morisi, 1970; Benazzi \& Gourbault, 1977; Bologna \& Vigna Taglianti, 1985).

Val Casotto: $114 \mathrm{Pi} / \mathrm{CN}$ Tana del Forno (pozzo o grotta dell'Orso) (Pamparato, frazione Serra) m 1045 (Morisi, 1972b; Bologna \& Vigna Taglianti, 1985). COROTIPO: Turanico-Europeo (TUE).

24. Nebria (Nebria) tibialis tibialis (Bonelli, 1810) Val Corsaglia: Bottero (Corsagliola) m 600 (LB). Val Casotto: Tetti Casotto (San Michele Mondovì) $\mathrm{m}$ 500 (LB); Ponte Tetti Casotto (San Michele Mondovì) m 500 (LB); Pamparato m 750 (LB); Castello di Casotto m 1050 (LB); Colla di Casotto m 1450 (LB \& PG).

COROTIPO: Alpino-Appenninico (ALAP).

25. Nebria (Boreonebria) rufescens (Stroem, 1768)

Val Casotto: Castello di Casotto m 1050 (LB).

COROTIPO: Oloartico (OLA).

26. Nebria (Eunebria) jockischii Sturm, 1815

Val Corsaglia: Pizzo d'Ormea (Magistretti, 1965);

Monte Mondolè (Magistretti, 1965).

Val Casotto: Castello di Casotto m 1050 (LB).

Corotipo: Centroeuropeo (CEU).

27. Nebria (Eunebria) picicornis (Fabricius, 1792) Val Corsaglia: Moline (Torre Mondovì) m 500 (LB). COROTIPO: Europeo (EUR).

28. Nebria (Eunebria) psammodes (P. Rossi, 1792) Val Corsaglia: Moline (Torre Mondovì) m 500 (LB); Bottero (Corsagliola) m 600 (LB).

Val Casotto: Ponte Tetti Casotto (San Michele Mondovì) $\mathrm{m} 500$ (LB).

Corotipo: S-Europeo (SEU).

29. Oreonebria (Oreonebria) ligurica (K. Daniel, 1903)

Val Corsaglia: Bocchin d'Aseo (Magistretti, 1965); Lago Revelli m 2000 (Bisio, 2008); Laghi della Brignola m 2200 (Bisio, 2008).

Corotipo: SW-Alpino (ALSW).

30. Oreonebria (Oreonebria) macrodera (K. Daniel, 1903) 
Val Corsaglia: Lago Revelli m 2000 (Bisio, 2008); Sella Brignola m 1900-2000 (LB \& PG); Laghi della Brignola m 2000 (Bisio, 2008); Monte Mondolè m 2000 (Bisio, 2008).

Val Casotto: Pizzo di Ormea (Magistretti, 1965). COROTIPO: Alpino-Appenninico (ALAP).

\section{Oreonebria (Oreonebria) angusticollis ${ }^{2}$ (Bo-} nelli, 1810)

Val Corsaglia: Bocchin d'Aseo (Magistretti, 1965: sub microcephala); Bocchin d'Aseo m 2200-2300 (Bisio, 2007); Bric Conoja m 2400 (Bisio, 2007); Lago Revelli m 2000 (Bisio, 2007); Laghi della Brignola $\mathrm{m} 2100$ (Bisio, 2007); Monte Mondolè $\mathrm{m} 2000$ (Bisio, 2007).

COROTIPO: W-Alpino (ALPW).

32. Notiophilus aquaticus (Linné, 1758)

Val Corsaglia: Pizzo di Ormea (Magistretti, 1965).

COROTIPO: Oloartico (OLA).

33. Notiophilus aestuans Dejean, 1826

Val Corsaglia: Pizzo d'Ormea (Magistretti, 1965: sub pusillus).

COROTIPO: Europeo (EUR).

34. Notiophilus palustris (Duftschmid, 1812)

Val Corsaglia: Montaldo Mondovì m 800 (LB).

COROTIPO: Sibirico-Europeo (SIE).

35. Elaphrus (Neoelaphrus) uliginosus Fabricius, 1792

Val Casotto: Pamparato m 750 (LB).

COROTIPO: Asiatico- Europeo (ASE).

36. Trechus (Trechus) quadristriatus (Schrank, 1781)

Val Casotto: Ponte Tetti Casotto (San Michele Mondovì) $\mathrm{m} 500$ (LB).

COROTIPO: Turanico-Europeo-Mediterraneo (TEM).

37. Trechus nicoleae Moncoutier, $1986^{3}$

Val Casotto: Val Casotto (Casale et al., 2006); Ca-

Sensu Bisio, 2007.

Sensu Magrini \& Degiovanni, 2012.

Sensu Degiovanni \& Magrini, 2016. stello di Casotto m 1050 (LB); Monte Berlino m 1700 (LB).

COROTIPO: SW-Alpino (ALSW).

38. Trechus liguricus Jeannel, 1921

Valle Mongia: $599 \mathrm{Pi} / \mathrm{CN}$ Grotta Oggeri (Bric Blin, Lisio) (Casale et al., 2019).

CoRotipo: SW-Alpino (ALSW).

39. Trechus maritimus Sainte-Claire Deville, 1907 Val Corsaglia: Bocchin d'Aseo (Magistretti, 1965). Bocchin d'Aseo m 2400 (Casale et al., 2006); Bric Conoia m 2300-2500 (Casale et al., 2006).

COROTIPO: SW-Alpino (ALSW).

40. Trechus fairmairei Pandellé, $1867^{4}$

Val Corsaglia: $993 \mathrm{Pi} / \mathrm{CN}$ Pozzo delle Pedane (Montaldo Mondovì) (Lana et al., 2014).

COROTIPO: Alpino-Appenninico (ALAP)

41. Duvalius carantii (Sella, 1874)

Val Corsaglia: Monte Mondolè, pozzo Cl, m 1870 c.a., presso il Buco dell'Artesinera n. $197 \mathrm{Pi} / \mathrm{CN}$ (Casale \& Vigna Taglianti, 1993); 3002 Pi/CN Buco di Roccia Bianca (Frabosa Soprana) (Lana et al., 2018); 967 Pi/CN Buco B di Roccia Bianca (Frabosa Soprana) (Lana et al., 2018).

Stenoendemita delle Alpi Liguri e delle Marittime, $D$. carantii è diffuso dalla Valle Stura di Demonte alla sinistra orografica dell'alta Val Corsaglia dove è presente solo marginalmente (per la distribuzione complessiva di $D$. carantii, D. lanai, D.morisii e $D$. chestai cfr. cartine-areale in Casale \& Vigna Taglianti, 1993, in Casale \& Giachino, 2010 e in Casale et al., 2019).

COROTIPO: SW-Alpino (ALSW).

42. Duvalius lanai Casale \& Giachino, 2010

Val Corsaglia: 3015 Pi/CN Grotta del Rospo (Sant'Anna Collarea, Montaldo Mondovì) (Casale \& Giachino, 2010); $201 \mathrm{Pi} / \mathrm{CN}$ Pozzo Congiuntivite (Sant'Anna Collarea, Montaldo Mondovi) (Casale \& Giachino, 2010).

D. lanai è uno stenoendemita della Val Corsaglia che, secondo Casale \& Giachino (2010), è particolarmente affine a $D$. carantii del quale è vicariante orientale con le caratteristiche di un elemento isolato periferico. Dalla più vicina stazione di quest'ultima specie è separato dal solco del Torrente Corsaglia, mentre il Rio 
Roburentello, che ne è il tributario, lo divide dalle località dove è presente $D$. morisii.

COROTIPO: SW-Alpino (ALSW).

43. Duvalius morisii Vigna Taglianti \& Casale, 1973

Val Casotto: $114 \mathrm{Pi} / \mathrm{CN}$ Tana del Forno (pozzo o grotta dell'Orso) (Pamparato, frazione Serra) m 1045 (Vigna Taglianti \& Casale, 1973, Morisi, 1973b; Bologna \& Vigna Taglianti, 1982, 1985); 115 Pi/CN Tana delle Turbiglie (Pamparato, frazione Serra) m 983 (Bologna \& Vigna Taglianti, 1985); $117 \mathrm{Pi} / \mathrm{CN}$ Tana della Fornace, m 1125 (Casale \& Giachino, 2010); 288 $\mathrm{Pi} / \mathrm{CN}$ Tana della Volpe m 1135 (Casale \& Giachino, 2010).

Valle Mongia: $884 \mathrm{Pi} / \mathrm{CN}$ Grotta di Rio dei Corvi (Bric del Fieno, Lisio) (Casale et al., 2019).

Stenoendemita della Val Casotto e della Valle Mongia, D. morisii è un'entità del gruppo di "Duvalius carantii" maggiomente affine a D. iulianae Vigna Taglianti $\&$ Casale,1973 del massiccio del Monte Saccarello. Corotipo: SW-Alpino (ALSW).

44. Duvalius chestai Casale, Giachino \& Lana, 2019 Valle Mongia: $599 \mathrm{Pi} / \mathrm{CN}$ Grotta Oggeri (Bric Blin, Lisio) (Casale et al., 2019).

D. chestai è uno stenoendemita noto unicamente della grotta citata. Per maggiori dettagli cfr. Casale et al. (2019).

COROTIPO: SW-Alpino (ALSW).

45. Scotodipnus glaber glaber Baudi di Selve, 1859

Val Casotto: Val Casotto (Baudi di Selve, 1871, 1890; Ganglbauer, 1900; Binaghi, 1936); Pamparato (Giachino, 1993).

COROTIPO: SW-Alpino (ALSW).

46. Tachyura (Tachyura) sexstriata (Duftschmid, 1812)

Val Casotto: Ponte Tetti Casotto (San Michele Mondovì) $\mathrm{m} 500$ (LB).

COROTIPO: Europeo (EUR).

Sensu Neri et al. (2013).

Sensu Vigna Taglianti (com. pers. 2011).
47. Asaphidion austriacum Schweiger, 1975

Val Corsaglia: San Giacomo di Roburent m 800 (LB). Corotipo: Centroeuropeo (CEU).

48. Bembidion (Metallina) lampros (Herbst, 1784) Val Corsaglia: Moline (Torre Mondovì) m 500 (LB); Rifugio Balma m 1900 (LB \& GA); Pizzo d'Ormea (Magistretti, 1965).

Val Casotto: Valcasotto m 950 (LB); Colla di Casotto m 1350 (LB); Monte Berlino m 1500-1700 (LB).

CoRotiPo: Paleartico (PAL).

49. Bembidion (Bembidion) quadrimaculatum (Linné, 1761)

Val Corsaglia: San Giacomo di Roburent m 800 (LB); San Rocco (Bossea) m 750 (LB).

Val Casotto: Ponte Tetti Casotto (San Michele Mondovì) $\mathrm{m} 500$ (LB); Ponte Desnì (Monasterolo Casotto) m 600 (LB); Pamparato m 800 (LB); Valcasotto m 950 (LB).

COROTIPO: Oloartico (OLA).

50. Bembidion (Trepanes) articulatum (Panzer, 1796)

Val Corsaglia: San Rocco (Bossea) m 750 (LB).

COROTIPO: Asiatico-Europeo (ASE).

51. Bembidion (Princidium) punctulatum Drapiez, 1820

Val Casotto: Ponte Desnì (Monasterolo Casotto) $\mathrm{m}$ 600 (LB); Pamparato m 800 (LB).

CoRotipo: Centroasiatico-Europeo-Mediterraneo (CEM).

52. Bembidion (Testedium) bipunctatum bipunctatum (Linné, 1761) ${ }^{5}$

Val Corsaglia: Laghi della Brignola m 2150 (LB \& GA); Monte Mondolè (Magistretti, 1965).

Corotipo: W-Paleartico (WPA) ${ }^{6}$.

53. Bembidion (Bembidionetolitzkya) varicolor (Fabricius, 1803)

Val Corsaglia: Bottero (Corsagliola) m 600 (LB); Corsaglia m 700 (LB); San Rocco (Bossea) m 750 (LB).

Val Casotto: Ponte Tetti Casotto (San Michele Mondovì) $\mathrm{m} 500$ (LB); Ponte Desnì (Monasterolo Casotto) m 600 (LB); Valcasotto m 950 (LB).

COROTIPO: Europeo (EUR). 
54. Bembidion (Bembidionetolitzkya) ascendens K. Daniel, 1902

Val Casotto: Pamparato m 800 (LB); Valcasotto m 950 (LB).

COROTIPO: Centroeuropeo (CEU).

55. Bembidion (Bembidionetolitzkya) geniculatum geniculatum Heer, 1837

Val Corsaglia: Sella Brignola m 1900 (LB \& PG).

Val Casotto: Ponte Desnì (Monasterolo Casotto) $\mathrm{m}$ 600 (LB); Pamparato m 800 (LB); Valcasotto m 950

(LB); Castello di Casotto m 1050 (LB).

COROTIPO: Europeo (EUR).

56. Bembidion (Bembidionetolitzkya) tibiale (Duftschmid, 1812)

Val Corsaglia: Corsaglia m 700 (LB); San Rocco (Bossea) m 750 (LB); Monte Mondolè (Magistretti, 1965).

Val Casotto: Ponte Desnì (Monasterolo Casotto) $\mathrm{m}$ 600 (LB); Pamparato m 800 (LB); Valcasotto m 950 (LB).

COROTIPO: Europeo (EUR).

57. Bembidion (Peryphiolus) monticola Sturm, 1825

Val Corsaglia: San Rocco (Bossea) m 750 (LB).

Val Casotto: Ponte Tetti Casotto (San Michele Mondovì) $\mathrm{m} 500$ (LB); Ponte Desnì (Monasterolo Casotto) m 600 (LB); Ponte Lisetto (Pamparato) m 700 (LB). COROTIPO: Europeo (EUR).

58. Bembidion (Nepha) genei illigeri Netolitzky, 1914

Val Casotto: Ponte Desnì (Monasterolo Casotto) $\mathrm{m}$ 600 (LB).

COROTIPO: Europeo-Mediterraneo (EUM).

59. Bembidion (Nepha) schmidti jeannelianum (Casale \& Vigna Taglianti, 1993)

Val Corsaglia: Monte Mondolè (Magistretti, 1965: sub schmidti jeanneli).

COROTIPO: Mediterraneo (MED).

60. Bembidion (Testediolum) jacqueti jacqueti (Jeannel, 1941)

Val Casotto: Monte Antoroto m 1800-2100 (Bisio, 2009b).

Val Corsaglia: Bochin d'Aseo m 2100-2200 (Bisio, 2009b: sub Ocydromus); Pizzo d'Ormea (De Monte, 1946); Pizzo d'Ormea m 2300 (Bisio, 2009b: sub Ocydromus); Cima Ciuaiera m 1700 (Bisio, 2009b: sub Ocydromus); Rifugio Balma (Monte Mondolè) $\mathrm{m}$ 1800 (Bisio, 2009b: sub Ocydromus); Laghi della Brignola $\mathrm{m} 2150$ (LB \& GA).

COROTIPO: Alpino-Appenninico (ALAP).

61. Bembidion (Testediolum) magellense alpicola (Jeannel, 1941)

Val Corsaglia: Bochin d'Aseo m 2100-2200 (Bisio, 2009b: sub Ocydromus); Bric Conoja m 2300-2500 (Bisio, 2009b: sub Ocydromus).

COROTIPO: Alpino-Appenninico (ALAP).

62. Bembidion (Ocydromus) decorum decorum (Zenker in Panzer, 1799)

Val Corsaglia: Bottero (Corsagliola) m 600 (LB); Corsaglia m 700 (LB); San Rocco (Bossea) m 750 (LB).

Val Casotto: Ponte Tetti Casotto (San Michele Mondovì) $\mathrm{m} 500$ (LB); Ponte Desnì (Monasterolo Casotto) m 600 (LB); Ponte Lisetto (Pamparato) $\mathrm{m} 700$ (LB); Pamparato m 800 (LB); Valcasotto m 950 (LB).

Corotipo: Centroasiatico-Europeo (CAE).

63. Bembidion (Peryphus) tetracolum Say, 1823

Val Corsaglia: Moline (Torre Mondovì) m 500 (LB); Corsaglia m 700 (LB); San Rocco (Bossea) m 750 (LB).

Val Casotto: Ponte Tetti Casotto (San Michele Mondovì) m 500 (LB); Ponte Scuole (Monasterolo Casotto) m 650 (LB); Pamparato m 800 (LB); Valcasotto m 950 (LB).

COROTIPO: Paleartico (PAL).

64. Bembidion (Peryphus) incognitum G. Müller, 1931

Val Corsaglia: Sella Brignola m 1900 (LB \& PG); Monte Mondolè (Magistretti, 1965).

Corotipo: Centroeuropeo (CEU).

65. Bembidion (Peryphanes) deletum Audinet-Serville, 1821

Val Casotto: Ponte Tetti Casotto (San Michele Mondovì) $\mathrm{m} 500$ (LB).

Val Corsaglia: San Giacomo di Roburent m 800 (LB). COROTIPO: Europeo (EUR). 
66. Bembidion (Peryphanes) italicum De Monte, 1943

Val Casotto: Ponte Tetti Casotto (San Michele Mondovì) $\mathrm{m} 500$ (Bisio, 2019).

Val Corsaglia: Rifugio Balma m 1900 (Bisio, 2019). COROTIPO: S-Europeo (SEU).

67. Sinechostictus (Sinechostictus) decoratus (Duftschmid, 1812)

Val Casotto: Ponte Desnì (Monasterolo Casotto) m 600 (LB).

Corotipo: Centroeuropeo (CEU).

68. Sinechostictus (Sinechostictus) ruficornis (Sturm, 1825)

Val Corsaglia: Moline (Torre Mondovì) m 500 (LB); Corsaglia m 700 (LB).

Val Casotto: Valcasotto m 950 (LB); Castello di Casotto m 1050 (LB).

Corotipo: Centroeuropeo (CEU).

69. Sinechostictus (Pseudolimnaeum) doderoi (Ganglbauer, 1891)

Val Casotto: Pamparato m 800, 1 es. 30.VI.2017 (LB).

Specie diffusa su Alpi e Appennino settentrionale, $S$. doderoi è un elemento ripicolo che si rinviene lungo i torrenti in ambienti forestali (Pesarini \& Monzini, 2011). Lungo l'arco alpino occidentale, di S. doderoi si conoscono poche stazioni. Oltre che nelle Alpi Liguri, dove è noto anche dellaVal Pesio (Magistretti, 1965; Ravizza, 1972; Bisio et al., 2013), esso è stato rinvenuto nelle Cozie (Bisio \& Giuntelli, 2011, 2014), nelle Pennine (Magistretti, 1965; Ravizza, 1972; Casale \& Vigna Taglianti, 1993) e nelle Lepontine occidentali (Magistretti, 1965).

Corotipo: Centroeuropeo (CEU).

70. Sinechostictus (Pseudolimnaeum) inustus (Jacquelin du Val, 1857)

Val Corsaglia: San Giacomo di Roburent m 800, 1 es. 15.V.2017.

S. inustus è ormai noto di diverse stazioni del Piemonte e della Valle d'Aosta (Allegro, 2014; Allegro \& Cersosimo, 2004; Allegro et al., 2002; Bisio, 2001; Bisio \& Giuntelli, 2006, 2014; Bisio et al., 2015a; Della Beffa, 1911; Ravizza, 1972), anche se la scarsità di reperti sembra confermare una sua generale sporadicità. A causa di tale scarsità la sua ecologia è ancora poco nota (cfr. Bonavita \& Vigna Taglianti, 2005). Infatti, come già evidenziato da uno degli autori (Bisio, 2009a), la specie si rinviene in biotopi alquanto diversi. Spesso è ripicola (cfr. Allegro, 2014; Allegro \& Cersosimo, 2004; Allegro et al., 2002; Bisio \& Giuntelli, 2006, 2014; Bisio et al., 2015; Müller, 1926; Ravizza, 1972), come nel caso dell'esemplare rinvenuto in Val Corsaglia. Talvolta fa invece registrare una spiccata sinantropia e si rinviene all'interno di cantine, nelle fessure di vecchi muri, tra i ruderi di vecchie baite e su suoli ammoniacali (cfr. Bisio, 2001; Bisio \& Giuntelli, 2014; Binaghi, 1935; Magistretti, 1965; Schatzmayr, 1942; Straneo, 1933, 1935). Vigna Taglianti (1982) ne evidenzia inoltre una certa troglofilia. È specie nuova per le Alpi Liguri.

COROTIPO: Centroeuropeo (CEU).

71. Abacetus (Astigis) salzmanni (Germar, 1824)

Val Casotto: Ponte Desnì (Monasterolo Casotto) $\mathrm{m}$ 600 (LB).

Entità ripicola e termofila, A. salzmanni è stata segnalata da Magistretti (1965) di diverse stazioni di Sicilia e di Sardegna, di alcune liguri e di un'unica località piemontese. In tempi più recenti, oltre che nella stazione citata, la specie è stata osservata in Piemonte lungo i Torrenti Scrivia (Casale et al., 2006: sub Astigis salzmanni), Orba (Casale et al., 2006: sub Astigis salzmanni; Allegro et al., 2004), Bormida (Allegro, 2014), Tanaro (Bisio \& Giuntelli, 2019) e Ticino (Monzini, 1986).

CoRotipo: W-Mediterraneo (WME).

72. Stomis (Stomis) pumicatus (Panzer, 1796)

Val Casotto: Tetti Casotto (San Michele Mondovì) $\mathrm{m}$ 500 (LB); Ponte Scuole (Monasterolo Casotto) m 650 (LB).

COROTIPO: Europeo (EUR).

73. Stomis (Stomis) elegans Chaudoir, 1861

Val Corsaglia: Laghi della Brignola m 2150 (LB \& GA); Monte Mondolè (Magistretti, 1965); Monte Mondolè $\mathrm{m} 1900$ (LB \& PG).

COROTIPO: W-Alpino (ALPW).

74. Poecilus (Poecilus) cupreus (Linné, 1758)

Val Corsaglia: Corsaglia m 700 (LB).

Corotipo: Asiatico-Europeo (ASE).

75. Poecilus (Poecilus) versicolor (Sturm, 1824) 
Val Corsaglia: San Giacomo di Roburent $\mathrm{m} 800$ (LB); Pizzo d'Ormea (Magistretti, 1965: sub Pterostichus coerulescens); Monte Mondolè (Magistretti, 1965: sub coerulescens).

Val Casotto: Val Casotto (Schatzmayr, 1930); Valcasotto m 950 (LB).

Corotipo: Asiatico-Europeo (ASE).

76. Poecilus (Macropoecilus) lepidus gressorius (Dejean, 1828)

Val Corsaglia: Corsaglia m 700 (LB).

Val Casotto: Ponte Tetti Casotto (San Michele Mondovì) m 500 (LB); Valcasotto m 950 (LB).

Corotipo: Sibirico-Europeo (SIE).

77. Pterostichus (Phonias) strenuus (Panzer, 1796) Val Casotto: Valcasotto m 950 (LB); Colla di Casotto m 1350 (LB \& PG).

Corotipo: Asiatico-Europeo (ASE).

78. Pterostichus (Platysma) niger (Schaller, 1783) Val Casotto: Tetti Casotto (San Michele Mondovì) $\mathrm{m}$ 500 (LB); Ponte Tetti Casotto (San Michele Mondovì) m 500 (LB).

Corotipo: Asiatico-Europeo (ASE).

79. Pterostichus (Pseudomaseus) nigrita (Paykull, 1790)

Val Casotto: Val Casotto (Schatzmayr, 1930).

Corotipo: Paleartico (PAL).

80. Pterostichus (Pseudomaseus) rhaeticus Heer, 1837

Val Corsaglia: Val Corsaglia (Casale et al., 2006).

Val Casotto: Tetti Casotto (San Michele Mondovi) $\mathrm{m}$ 500 (LB); Valcasotto m 950 (LB); Castello di Casotto m 1050 (LB); Colla di Casotto m 1450 (LB \& PG).

COROTIPO: Europeo (EUR).

81. Pterostichus (Pseudorites) nicaeensis (A. Villa \& G.B. Villa, 1835)

Val Casotto: Ponte Scuole (Monasterolo Casotto) $\mathrm{m}$ 650 (LB); Castello di Casotto m 1050 (LB); Colla di Casotto m 1450 (LB \& PG).

Corotipo: SW-Alpino (ALSW).

82. Pterostichus (Platypterus) truncatus dilatatus (A. Villa \& G.B. Villa, 1835)

Val Corsaglia: Pizzo d'Ormea (Magistretti, 1965);
Sella Brignola m 1900-2000 (LB \& PG); Monte Mondolè (Magistretti, 1965); Monte Mondolè $\mathrm{m} 1900$ (LB \& $\mathrm{PG})$.

Val Casotto: Monte Berlino m 1700 (LB \& PG).

COROTIPO: W-Alpino (ALPW).

83. Pterostichus (Pterostichus) impressicollis (Fairmaire \& Laboulbène, 1854)

Val Corsaglia: Bottero (Corsagliola) m 600 (LB); Bocchin d'Aseo (Capra, 1941); Bocchin d'Aseo m 2294 (Giachino \& Casale, 1983); Bric Conoja m 1600 (Giachino \& Casale, 1983); Sella Brignola m 19002000 (LB \& GA); Laghi della Brignola m 2150 (LB \& GA).

Val Casotto: Pamparato m 900 (LB); Castello di Casotto (Giachino \& Casale, 1983; Castello di Casotto m 1050 (LB); Colla di Casotto (Capra, 1941); Monte Berlino m 1500-1700 (LB); Balma del Mondolè (Giachino \& Casale, 1983).

COROTIPO: SW-Alpino (ALSW).

84. Pterostichus (Pterostichus) funestes Csiki, 1930

Val Corsaglia: Montaldo Mondovì m 800 (LB); Bocchin d'Aseo (Schatzmayr, 1930); Monte Conoja (Sciaky, 1984); Pizzo d'Ormea (Schatzmayr, 1930); Monte Mondolè (Magistretti, 1965: sub stipanovichi). Val Casotto: Tetti Casotto (San Michele Mondovi) m 500 (LB); Ponte Tetti Casotto (San Michele Mondovi) m 500 (LB); Pamparato m 900 (LB); 114 Pi/CN Tana del Forno (pozzo o grotta dell'Orso) (Pamparato, frazione Serra) m 1045 (Morisi, 1972b: sub cristatus; Bologna \& Vigna Taglianti, 1985); Valcasotto m 950 (LB); Castello di Casotto (Sciaky, 1984); Colla di Casotto (Sciaky, 1984); Colla di Casotto m 1350 (LB \& PG); Monte Berlino m 1500-1700 (LB \& PG).

COROTIPO: SW-Alpino (ALSW).

85. Pterostichus (Oreophilus) morio liguricus J. Daniel, 1903

Val Corsaglia: Laghi della Brignola 2100-2300 m (Bisio, 1995); Laghi della Brignola m 2150 (LB \& GA); Monte Mondolè (Magistretti, 1965); Monte Mondolè $\mathrm{m} 2100 \mathrm{~m}$ (Bisio, 1995).

Val Casotto: Colla di Casotto (Casale et al., 2006); Colla di Casotto m 1350 (LB); Monte Berlino m 15001700 (LB \& PG); Monte Mussiglione m 1950 (LB \& $\mathrm{PG})$.

CoRotipo: Centroeuropeo (CEU). 
86. Pterostichus (Oreophilus) bicolor bicolor Aragona, 1830

Val Corsaglia: Bocchin d'Aseo (Schatzmayr, 1930); Laghi della Brignola m 2100 (LB \& PG); Monte Mondolè (Magistretti, 1965); Monte Mondolè m 1900 (LB $\& \mathrm{PG})$.

Val Casotto: Valcasotto m 950 (LB); Colla di Casotto (Casale et al., 2006); Colla di Casotto m 1350 (LB); Monte Berlino m 1500-1700 (LB \& PG). Corotipo: Alpino-Appenninico (ALAP).

87. Pterostichus (Oreophilus) yvanii (Dejean, 1828)

Val Corsaglia: Monte Mondolè (Magistretti, 1965).

COROTIPO: W-Alpino (ALPW).

88. Molops ovipennis medius Chaudoir, 1868

Val Corsaglia: San Giacomo di Roburent m 1200 (Casale et al., 2006).

Val Casotto: $114 \mathrm{Pi} / \mathrm{CN}$ Tana del Forno (pozzo o grotta dell'Orso) (Pamparato, frazione Serra) m 1045 (Morisi, 1972b; Bologna \& Vigna Taglianti, 1985).

COROTIPO: Alpino-Appenninico (ALAP).

89. Abax (Abax) parallelepipedus ligurinus Zanella, 2017

Val Casotto: Colla di Casotto m 1450 (LB \& PG).

COROTIPO: Europeo (EUR).

90. Abax (Abax) contractus (Heer, 1841)

Val Corsaglia: Bottero (Corsagliola) $\mathrm{m} 600$ (LB); San Rocco (Bossea) m 700 (LB); Montaldo Mondovì m 800 (LB).

Val Casotto: Tetti Casotto (San Michele Mondovì) m 500 (LB); Ponte Tetti Casotto (San Michele Mondovì) m 500 (LB); 114 Pi/CN Tana del Forno (pozzo o grotta dell'Orso) (Pamparato, frazione Serra) m 1045 (Morisi, 1972b: sub continuus; Bologna \& Vigna Taglianti, 1985: sub continuus).

COROTIPO: S-Alpino (ALPS).

91. Amara (Zezea) fulvipes (Audinet-Serville, 1821)

Val Casotto: Pamparato m 900 (LB).

COROTIPO: Europeo (EUR).

Sensu Zanella (2017).
92. Amara (Amara) aenea (De Geer, 1774)

Val Corsaglia: Moline (Torre Mondovì) m 500 (LB); San Giacomo di Roburent m 800 (LB).

Val Casotto: Ponte Tetti Casotto (San Michele Mondovì) $\mathrm{m} 500$ (LB); Pamparato m 900 (LB); Colla di Casotto m 1350 (LB); Monte Berlino m 1500-1700 (LB \& PG).

COROTIPO: Paleartico (PAL).

93. Amara (Amara) convexior Stephens, 1828

Val Casotto: Pamparato m 900 (LB); Valcasotto m 950 e 1000 (LB).

Corotipo: Sibirico-Europeo (SIE).

94. Amara (Amara) curta Dejean, 1828

Val Casotto: Ponte Tetti Casotto (San Michele Mondovì) $\mathrm{m} 500$ (LB); Monte Berlino m 1700-1800 (LB $\& \mathrm{PG})$.

Corotipo: Sibirico-Europeo (SIE).

95. Amara (Amara) eurynota (Panzer, 1796)

Val Corsaglia: Sella Brignola m 1900 (LB \& PG). Corotipo: Sibirico-Europeo (SIE).

96. Amara (Amara) familiaris (Duftschmid, 1812) Val Corsaglia: San Giacomo di Roburent m 800 (LB); Val Casotto: Ponte Tetti Casotto (San Michele Mondovi) $\mathrm{m} 500$ (LB).

Corotipo: Sibirico-Europeo (SIE).

97. Amara (Amara) lunicollis Schiödte, 1837

Val Corsaglia: Pizzo d'Ormea (Magistretti, 1965).

Val Casotto: Valcasotto m 950 (LB).

COROTIPO: Oloartico (OLA).

98. Amara (Amara) nitida Sturm, 1825

Val Corsaglia: Rifugio Balma m 1900 (LB \& GA).

Val Casotto: Pamparato m 900 (LB); Colla di Casotto m 1350 (LB); Monte Berlino m 1500-1700 (LB). COROTIPO: Asiatico-Europeo (ASE).

99. Amara (Amara) ovata (Fabricius, 1792)

Val Corsaglia: San Giacomo di Roburent m 800 (LB); Monte Mondolè (Magistretti, 1965).

Val Casotto: Ponte Tetti Casotto (San Michele Mondovì) $\mathrm{m} 500$ (LB).

Corotipo: Asiatico-Europeo (ASE).

100. Amara (Amara) similata (Gyllenhal, 1810) 
Val Casotto: Ponte Tetti Casotto (San Michele Mondovì) $\mathrm{m} 500$ (LB).

COROTIPO: Asiatico-Europeo (ASE).

101. Amara (Celia) bifrons (Gyllenhal, 1810)

Val Corsaglia: Pizzo d'Ormea (Magistretti, 1965).

Val Casotto: Ponte Tetti Casotto (San Michele Mondovì) $\mathrm{m} 500$ (LB).

COROTIPO: Centroasiatico-Europeo (CAE).

102. Amara (Celia) erratica (Duftschmid, 1812)

Val Corsaglia: Laghi della Brignola m 2150 (LB \& GA); Monte Mondolè $\mathrm{m} 1900$ (LB \& PG).

COROTIPO: Oloartico (OLA).

103. Amara (Xenocelia) municipalis (Duftschmid, 1812)

Val Casotto: Ponte Tetti Casotto (San Michele Mondovì) $\mathrm{m} 500$ (LB).

Corotipo: Sibirico-Europeo (SIE).

104. Amara (Percosia) equestris equestris (Duftschmid, 1812)

Val Corsaglia: Pizzo d'Ormea (Hieke, 1978); Monte Mondolè (Hieke, 1978); Monte Mondolè m 1900 (Bisio, 2009c).

COROTIPO: Asiatico-Europeo (ASE).

105. Amara (Leiromorpha) lantoscana lantoscana Fauvel, 1888

Val Corsaglia: Pizzo di Ormea (K. Daniel, 1906; Luigioni, 1929).

CoRotipo: SW-Alpino (ALSW).

106. Amara (Leirides) cardui psyllocephala K. Daniel \& J. Daniel, 1898

Val Corsaglia: Bochin d'Aseo (Magistretti, 1965); Bochin d'Aseo m 2200 e a 2400 (Bisio, 2002); Pizzo d'Ormea (K. Daniel \& J. Daniel, 1898; Luigioni, 1929).

COROTIPO: W-Alpino (ALPW).

107. Chlaeniellus vestitus (Paykull, 1790)

Val Casotto: Ponte Desnì (Monasterolo Casotto) $\mathrm{m}$ 600 (LB); Pamparato m 800 (LB).

CoRotipo: Europeo-Mediterraneo (EUM).

108. Chlaenius velutinus velutinus (Duftschmid, 1812)
Val Casotto: Ponte Tetti Casotto (San Michele Mondovì) $\mathrm{m} 500$ (LB).

COROTIPO: Europeo-Mediterraneo (EUM).

109. Callistus lunatus (Fabricius, 1775)

Val Casotto: Tetti Casotto (San Michele Mondovì) $\mathrm{m}$ 500, 1 es. 5.V.2017 (LB).

Corotipo: Turanico-Europeo (TUE).

110. Licinus (Licinus) depressus (Paykull, 1790)

Val Corsaglia: Pizzo d'Ormea (Magistretti, 1965).

Elemento dalle spiccate esigenze xerotermofile, nelle

Alpi Occidentali $L$. depressus è noto di alcune località piemontesi (Ghiliani, 1887; Jeannel, 1942; Magistretti, 1965; Casale, 1977) e di molte stazioni valdostane (Casale, 1977; Bisio, 2015, dati inediti; Bisio et al., 2015c, 2016).

Corotipo: Asiatico-Europeo (ASE).

111. Badister (Badister) bullatus (Schrank, 1798)

Val Casotto: Tetti Casotto (San Michele Mondovì) $\mathrm{m}$ 500 (LB).

COROTIPO: Oloartico (OLA).

112. Anisodactylus (Anisodactylus) binotatus (Fabricius, 1787)

Val Casotto: Ponte Tetti Casotto (San Michele Mondovì) $\mathrm{m} 500$ (LB).

Val Corsaglia: Corsaglia m 700 (LB).

COROTIPO: Asiatico-Europeo (ASE).

113. Diachromus germanus (Linné, 1758)

Val Corsaglia: Moline (Torre Mondovì) m 500 (LB).

Val Casotto: Tetti Casotto (San Michele Mondovì) m 500 (LB); Ponte Tetti Casotto (San Michele Mondovi) m 500 (LB).

CoRotiPO: Turanico-Europeo-Mediterraneo (TEM).

114. Stenolophus teutonus (Schrank, 1781)

Val Corsaglia: Moline (Torre Mondovì) m 500 (LB); Bottero (Corsagliola) m 600 (LB).

Val Casotto: Tetti Casotto (San Michele Mondovì) $\mathrm{m}$ 500 (LB); Ponte Tetti Casotto (San Michele Mondovi) m 500 (LB).

COROTIPO: Turanico-Europeo-Mediterraneo (TEM).

115. Bradycellus (Bradycellus) verbasci (Duftschmid, 1812) 
Val Casotto: Ponte Tetti Casotto (San Michele Mondovì) $\mathrm{m} 500$ (LB).

COROTIPO: Turanico-Europeo (TUE).

116. Bradycellus (Bradycellus) caucasicus (Chaudoir, 1846)

Val Corsaglia: Pizzo d'Ormea (Magistretti, 1965: sub collaris; Jaeger, 2008).

Val Casotto: Colla di Casotto m 1350 (LB).

COROTIPO: Sibirico-Europeo (SIE).

117. Acupalpus (Acupalpus) flavicollis (Sturm, 1825)

Val Corsaglia: San Rocco (Bossea) m 750 (LB).

COROTIPO: Europeo (EUR).

118. Ophonus (Hesperophonus) cribricollis (Dejean, 1829)

Val Corsaglia: Moline (Torre Mondovì) m 500 (LB);

Roburent $\mathrm{m} 700$ (LB).

Val Casotto: Pamparato m 900 (LB).

COROTIPO: Turanico-Europeo (TUE).

119. Ophonus (Metophonus) puncticeps Stephens, 1828

Val Casotto: Ponte Tetti Casotto (San Michele Mondovì) $\mathrm{m} 500$ (LB).

COROTIPO: -Europeo (TUE).

120. Ophonus (Metophonus) rufibarbis (Fabricius, 1792)

Val Corsaglia: Corsaglia m 700 (LB).

COROTIPO: Turanico-Europeo-Mediterraneo (TEM).

121. Pseudoophonus (Pseudoophonus) rufipes (Degeer, 1774)

Val Corsaglia: Moline (Torre Mondovì) m 500 (LB). Val Casotto: Tetti Casotto (San Michele Mondovì) $\mathrm{m}$ 500 (LB); Ponte Tetti Casotto (San Michele Mondovì) m 500 (LB).

Corotipo: Paleartico (PAL).

122. Harpalus (Harpalus) affinis (Schrank, 1781)

Val Corsaglia: Corsaglia m 700 (LB).

Val Casotto: Castello di Casotto m 1050 (LB); Colla di Casotto m 1350 (LB).

Corotipo: Asiatico-Europeo (ASE).

123. Harpalus (Harpalus) distinguendus (Duftschmid, 1812)
Val Corsaglia: Moline (Torre Mondovì) m 500 (LB). Val Casotto: Ponte Tetti Casotto (San Michele Mondovì) $\mathrm{m} 500$ (LB).

COROTIPO: Paleartico (PAL).

124. Harpalus (Harpalus) dimidiatus (P. Rossi, 1790)

Val Corsaglia: Moline (Torre Mondovì) m 500 (LB); Roburent m 700 (LB); San Giacomo di Roburent m 800 (LB).

Val Casotto: Tetti Casotto (San Michele Mondovì) $\mathrm{m}$ 500 (LB); Castello di Casotto m 1050 (LB).

COROTIPO: Europeo (EUR).

125. Harpalus (Harpalus) rubripes (Duftschmid, 1812)

Val Corsaglia: Pizzo d'Ormea (Magistretti, 1965); Monte Mondolè (Magistretti, 1965).

Val Casotto: Ponte Tetti Casotto (San Michele Mondovì) $\mathrm{m} 500$ (LB); Pamparato m 900 (LB).

COROTIPO: Asiatico-Europeo (ASE).

126. Harpalus (Harpalus) laevipes Zetterstedt, 1828

Val Casotto: Monte Antoroto (Magistretti, 1965: sub quadripunctatus).

Corotipo: Asiatico-Europeo (ASE).

127. Harpalus (Harpalus) marginellus Dejean, 1829

Val Casotto: Monte Berlino m 1500-1700 (LB).

Corotipo: Centroeuropeo (CEU).

128. Harpalus (Harpalus) atratus Latreille, 1804

Val Corsaglia: Moline (Torre Mondovì) m 500 (LB); Montaldo Mondovì m 800 (LB).

Val Casotto: Ponte Tetti Casotto (San Michele Mondovì) $\mathrm{m} 500$ (LB).

COROTIPO: Europeo (EUR).

129. Harpalus (Harpalus) solitaris Dejean, 1829

Val Corsaglia: Laghi della Brignola m 2100 (LB \& PG).

Corotipo: Oloartico (OLA).

130. Harpalus (Harpalus) honestus (Duftschmid, 1812)

Val Casotto: Ponte Tetti Casotto (San Michele Mondovì) $\mathrm{m} 500$ (LB). 
Corotipo: Sibirico-Europeo (SIE).

131. Harpalus (Harpalus) serripes (Quensel in Schönherr, 1806)

Val Casotto: Tetti Casotto (San Michele Mondovì) $m$ 500 (LB).

COROTIPO: Paleartico (PAL).

132. Harpalus (Harpalus) tardus (Panzer, 1797)

Val Casotto: Tetti Casotto (San Michele Mondovì) m 500 (LB); Ponte Tetti Casotto (San Michele Mondovì) m 500 (LB); Pamparato m 800 (LB).

Val Corsaglia: Roburent m 700 (LB).

COROTIPO: Asiatico-Europeo (ASE).

133. Harpalus (Harpalus) anxius (Duftschmid, 1812) Val Corsaglia: Moline (Torre Mondovì) m 500 (LB); Roburent $\mathrm{m} 700$ (LB).

Val Casotto: Pamparato m 900 (LB).

COROTIPO: Paleartico (PAL).

134. Parophonus (Parophonus) maculicornis (Duftschmid, 1812)

Val Corsaglia: Moline (Torre Mondovì) m 500 (LB). COROTIPO: S-Europeo (SEU).

135. Parophonus (Parophonus) mendax (P. Rossi, 1790)

Val Casotto: Tetti Casotto (San Michele Mondovì) m 500 (LB).

Specie nuova per le Alpi Liguri. Nelle Alpi Occidentali P. mendax era sino a oggi noto solo della Val Maira (cfr. Bisio \& Giuntelli, 2011).

COROTIPO: S-Europeo (SEU).

136. Trichotichnus (Trichotichnus) laevicollis (Duftschmid, 1812)

Val Casotto: Monte Berlino m 1500-1700 (LB \& PG). COROTIPO: Centroeuropeo (CEU).

137. Trichotichnus (Trichotichnus) nitens (Heer, 1838)

Val Casotto: Colla di Casotto m 1450 (LB); Monte Berlino m 1500-1700 (LB \& PG).

Corotipo: Centroeuropeo (CEU).

138. Synuchus vivalis (Illiger, 1798)

Val Corsaglia: Pizzo d'Ormea (Magistretti, 1965: sub nivalis).
Corotipo: Asiatico-Europeo (ASE)

139. Calathus (Calathus) fuscipes graecus Dejean, 1831

Val Corsaglia: Moline (Torre Mondovì) m 500 (LB); Monte Mondolè (Magistretti, 1965).

Val Casotto: Tetti Casotto (San Michele Mondovì) $\mathrm{m}$ 500 (LB); Castello di Casotto m 1050 (LB).

COROTIPO: Europeo-Mediterraneo (EUM).

140. Calathus (Neocalathus) melanocephalus (Linné, 1758)

Val Corsaglia: Corsaglia m 700 (LB).

Val Casotto: Tetti Casotto (San Michele Mondovì) $\mathrm{m}$ 500 (LB); Castello di Casotto m 1050 (LB).

COROTIPO: Paleartico (PAL).

141. Calathus (Neocalathus) micropterus (Duftschmid, 1812)

Val Corsaglia: Monte Mondolè (Magistretti, 1965). COROTIPO: Oloartico (OLA).

142. Calathus (Neocalathus) erratus (C.R. Sahlberg, 1827)

Val Casotto: Ponte Tetti Casotto (San Michele Mondovì) $\mathrm{m} 500$ (LB).

COROTIPO: Sibirico-Europeo (SIE).

143. Sphodropsis ghilianii ghilianii (Schaum, 1858) Val Corsaglia: 3015 Pi/CN Grotta del Rospo (Sant'Anna Collarea, Montaldo Mondovì) (Lana et al., 2008; Casale \& Giachino, 2010); 112 Pi/CN Tana di San Luigi (= Grotta dello Spelerpes) (Roburent) $\mathrm{m}$ 780 (Morisi, 1970; Benazzi \& Gourbault, 1977; Bologna \& Vigna Taglianti, 1985); $113 \mathrm{Pi} / \mathrm{CN}$ Tana di Camplass (=Grotta di Campelasso, Grotta degli Assassini) (Roburent, Case Garian, loc. Camplass) m 810 (Müller, 1930; Wolf, 1937, 1938; Binaghi, 1939; Magistretti, 1965; Bordoni, 1968; Martinotti, 1968); 103 Pi/CN Grotta di Bossea m 836 (Baudi di Selve, 1890: sub Antisphodrus; Dellepiane, 1924; Müller, 1930; Binaghi, 1939; Magistretti, 1965; Bordoni, 1968; Martinotti, 1968; Morisi, 1969, 1971a, 1971b; Peano, 1973, 1974; Bologna \& Vigna Taglianti, 1985).

Val Casotto: $114 \mathrm{Pi} / \mathrm{CN}$ Tana del Forno (pozzo o grotta dell'Orso) (Pamparato, frazione Serra) m 1045 (Morisi, 1972b); 115 Pi/CN Tana delle Turbiglie (Pamparato, frazione Serra) m 983 (Vigna Taglianti \& Casale, 1973; Bologna \& Vigna Taglianti, 1985). 
Valle Mongia: $599 \mathrm{Pi} / \mathrm{CN}$ Grotta Oggeri (Bric Blin, Lisio) (Casale et al., 2019).

COROTIPO: W-Alpino (ALPW).

144. Laemostenus (Laemostenus) janthinus coeruleus (Dejean, 1828)

Val Corsaglia: Monte Mondolè (Magistretti, 1965).

Val Casotto: Monte Berlino m 1500-1700 (LB \& PG); Monte Mussiglione m 1950 (LB \& PG).

COROTIPO: S-Alpino (ALPS).

145. Laemostenus (Actenipus) obtusus (Chaudoir, 1861)

Val Corsaglia: $112 \mathrm{Pi} / \mathrm{CN}$ Tana di San Luigi (=Grotta dello Spelerpes) (Roburent) m 780 (Morisi, 1970, 1973a; Benazzi \& Gourbault, 1977; Bologna \& Vigna Taglianti, 1985); Montaldo Mondovì m 800 (LB).

COROTIPO: SW-Alpino (ALSW).

146. Agonum (Agonит) mиelleri muelleri (Herbst, 1784)

Val Casotto: Colla di Casotto m 1350 (LB).

Corotipo: Sibirico-Europeo (SIE).

147. Agonum (Melanagonum) viduum (Panzer, 1796)

Val Corsaglia: Bottero (Corsagliola) m 600 (LB); San Rocco (Bossea) m 750 (LB).

Val Casotto: Ponte Tetti Casotto (San Michele Mondovì) $\mathrm{m} 500$ (LB); Ponte Desnì (Monasterolo Casotto) m 600 (LB); Pamparato m 750 (LB).

COROTIPO: Sibirico-Europeo (SIE).

148. Agonum (Melanagonum) emarginatum (Gyllenhall, 1827)

Val Casotto: Pamparato m 750 (LB).

COROTIPO: Europeo (EUR).

149. Agonum (Punctagonum) sexpunctatum (Linné, 1758)

Val Casotto: Ponte Tetti Casotto (San Michele Mondovì) m 500 (LB); Valcasotto m 950 (LB); Colla di Casotto m 1350 (LB).

Corotipo: Sibirico-Europeo (SIE).

150. Anchomenus (Anchomenus) dorsalis (Pontoppidan, 1763)

Val Corsaglia: Moline (Torre Mondovì) m 500 (LB). Val Casotto: Tetti Casotto (San Michele Mondovì) m
500 (LB); Ponte Tetti Casotto (San Michele Mondovi) m 500 (LB); Valcasotto m 950 (LB); Colla di Casotto m 1350 (LB).

COROTIPO: Paleartico (PAL).

151. Platynus peirolerii (Bassi, 1834)

Val Corsaglia: Sella Brignola m 1900 (LB \& PG); Laghi della Brignola m 2100 (LB \& PG); Monte Mondolè (Magistretti, 1965); Monte Mondolè $\mathrm{m} 1900$ (LB $\& \mathrm{PG})$.

Val Casotto: Colla di Casotto m 1450 (LB \& PG); Monte Berlino m 1500-1700 (LB \& PG); Monte Mussiglione $\mathrm{m} 1950$ (LB \& PG).

COROTIPO: SW-Alpino (ALSW).

152. Limodromus assimilis (Paykull, 1790)

Val Corsaglia: Moline (Torre Mondovì) m 500 (LB); Bottero (Corsagliola) m 600 (LB).

Val Casotto: Tetti Casotto (San Michele Mondovì) $\mathrm{m}$ 500 (LB); Ponte Tetti Casotto (San Michele Mondovi) m 500 (LB); Pamparato m 750 (LB); Valcasotto m 950 (LB); Castello di Casotto m 1050 (LB); Colla di Casotto m 1350 (LB); Monte Berlino m 1500-1700 (LB $\& \mathrm{PG})$.

COROTIPO: Sibirico-Europeo (SIE).

153. Paranchus albipes (Fabricius, 1796)

Val Corsaglia: Bottero (Corsagliola) m 600 (LB); Corsaglia m 700 (LB).

Val Casotto: Ponte Lisetto (Pamparato) m 700 (LB). COROTIPO: Europeo-Mediterraneo (EUM).

154. Cymindis (Cymindis) humeralis (Geoffroy in Fourcroy, 1785)

Val Corsaglia: Pizzo d'Ormea (Magistretti, 1965); Laghi della Brignola m 2100 (LB \& PG); Monte Mondolè (Magistretti, 1965); Monte Mondolè $\mathrm{m} 1900$ (LB $\& \mathrm{PG})$.

COROTIPO: Europeo (EUR).

155. Syntomus truncatellus (Linné, 1761)

Val Corsaglia: Roburent m 700 (LB); San Rocco (Bossea) m 700 (LB).

Corotipo: Sibirico-Europeo (SIE).

156. Microlestes minutulus (Goeze, 1777)

Val Corsaglia: Corsaglia m 700 (LB).

Val Casotto: Pamparato m 900 (LB).

COROTIPO: Oloartico (OLA). 
157. Dromius (Dromius) agilis (Fabricius, 1787)

Val Casotto: Valcasotto m 1000 (LB).

Corotipo: Sibirico-Europeo (SIE).

158. Lamprias cyanocephalus (Linné, 1758)

Val Corsaglia: Pizzo di Ormea (Magistretti, 1965).

Corotipo: Paleartico (PAL).

159. Drypta dentata (P. Rossi, 1790)

Val Casotto: Tetti Casotto (San Michele Mondovì) m 500 (LB); Ponte Tetti Casotto (San Michele Mondovi) m 500 (LB).

Corotipo: Afrotropicale e Paleartico (AFP).

\section{Principali Carabidocenosi Rilevate}

Carabidocenosi silvicole delle fasce submontana $\mathrm{e}$ montana. Le carabidocenosi silvicole che popolano le foreste delle valli in oggetto hanno una composizione ricca e variegata e sono costituite, oltre che da entità a vasto areale, da diversi endemiti. Nelle formazioni boschive a castagno e miste dell'orizzonte submontano l'elemento silvicolo più comune è $A b a x$ contractus $^{8}$, diffuso dalle confluenze delle tre valli sino a una quota accertata di quasi $1100 \mathrm{~m}$. Altre entità più o meno comuni sintopiche con questa specie sono Carabus granulatus, C. intricatus, C. monticola, $\mathrm{Ne}$ bria brevicollis, N. tibialis, Notiophilus palustris, Stomis pumicatus, Pterostichus rhaeticus, P. funestes, $P$. impressicollis e Limodromus assimilis. Completano l'elenco delle specie silvicole di questo orizzonte Leistus spinibarbis e Stomis pumicatus, taxa che paiono molto meno frequenti.

Nella fascia montana le specie censite risultano molto più numerose. Infatti, ad alcuni elementi silvicoli della fascia submontana censiti anche in quella superiore si aggiunge un gruppo consistente di taxa, alcuni dei quali maggiormente legati alla fascia climacica del faggio. Ben rappresentati sono i generi Pterostichus ( $P$. rhaeticus, $P$. nicaeensis, $P$. truncatus, $P$. impressicollis, $P$. funestes, $P$. morio e $P$. bicolor) e $C a$ rabus (C. granulatus, C. monticola, $C$. glabratus, $C$. convexus, C. germari, C. solieri). Altre entità note della fascia montana sono Aptinus alpinus, Cychrus italicus, C. attenuatus, Nebria brevicollis, N. tibialis,

Sensu Zanella (2017).
Trechus nicoleae, Molops ovipennis, Abax parallelepipedus, Trichotichnus laevicollis, T. nitens, Synuchus vivalis, Calathus micropterus, Platynus peirolerii e $\mathrm{Li}$ modromus assimilis. Tendenzialmente molto meno comuni risultano invece Leistus ferrugineus e L. nitidus.

Carabidocenosi praticole delle fasce forestali. Anche la composizione delle carabidocenosi che popolano le formazioni aperte negli orizzonti submontano e montano è molto ricca e variegata, anche se la maggioranza dei reperti è stata ottenuta nella fascia submontana dove l'estensione della superficie occupata dalle formazioni aperte è molto maggiore.

Lungo i prati falciabili che occupano i suoli alluvionali di fondovalle della fascia del castagno è stata riscontrata la presenza abbondante di quattro specie di Brachinus (B. elegans, B. explodens, B. glabratus e $B$. sclopeta) osservate spesso in sintopia con Anchomenus dorsalis. Di questa fascia sono note anche numerose Harpalinae (Anisodactylus binotatus, Diachromus germanus, Stenolophus teutonus, Bradycellus verbasci, Acupalpus flavicollis, Ophonus cribricollis, $O$. puncticeps, O. rufibarbis, Pseudoophonus rufipes, Harpalus affinis, $H$. distinguendus, $H$. dimidiatus, $H$. rubripes, $H$. atratus, $H$. honestus, $H$. serripes, $H$. tardus, H. anxius, Parophonus maculicornis e P. mendax) e diverse Amara (A. fulvipes, A. aenea, A. convexior, A. curta, A. familiaris, A. lunicollis, A. nitida, A. ovata, A. similata, A. bifrons e A. municipalis). Altri Carabidi segnalati sono Tachyura sexstriata, Bembidion lampros, Poecilus cupreus, P. versicolor, P. lepidus, Pterostichus niger, Badister bullatus, Calathus fuscipes, C. melanocephalus, C. erratus, Agonum viduum, A. emarginatum, A. sexpunctatum, Syntomus truncatellus, Microlestes minutulus e Drypta dentata. Molto più raro risulta invece Callistus lunatus.

Nell'orizzonte montano le ricerche - effettuate soprattutto alla Colla di Casotto, nei prati di ridotta estensione che circondano il Castello di Casotto e in alcune radure erbose che interrompono qua e là la continuità della foresta - hanno dato risultati più modesti; sono quindi relativamente poche le specie che vanno ad aggiungersi a quelle già note dalla letteratura. L'entità di maggior pregio faunistico nota è Licinus depressus, entità xerotermofile segnalata da Magistretti (1965) per il Pizzo d'Ormea. Le altre specie praticole note di questa fascia sono invece tutte entità più $o$ meno comuni: Cicindela campestris, Carabus problematicus, Bembidion lampros, Poecilus versicolor, Pte- 
rostichus strenuus, Amara aenea, A. curta, A. eurynota, A. nitida, A. equestris, Bradycellus caucasicus, Harpalus affinis, H. marginellus, Calathus fuscipes $C$. melanocephalus, Laemostenus janthinus, Agonum muelleri e A. sexpunctatum.

Carabidocenosi cripticole. Il sistema di microfessure dell'ambiente sotterraneo superficiale (M.S.S.) della Val Corsaglia, della Val Casotto e della Val Mongia è popolato da carabidocenosi cripticole di notevole interesse. In particolare, sono da ricordare per il loro elevato pregio faunistico, quattro entità ipogeobie (sensu Giachino \& Vailati, 2016) rinvenute in diverse grotte del territorio in oggetto: Duvalius carantii, D. lanai, D. morisii e D. chestai. La prima specie è nota di alcune cavità in quota del Monte Mondolè e di due grotte di Frabosa Soprana dove pare raggiungere il limite orientale del suo areale. D. lanai, entità affine a D. carantii del quale è vicariante orientale a Est del solco del Corsaglia, è stato rinvenuto in due grotte nel Comune di Montaldo Mondovì. D. morisii - taxon maggiomente affine a $D$. iulianae Vigna Taglianti \& Casale, 1973 del massiccio del Monte Saccarello - è noto, ancora più a Est, di alcune grotte della Val Casotto e di una grotta della Val Mongia. Infine, D. chestai è stato rinvenuto di recente all'interno della Grotta Oggeri in quest'ultima valle (per la distribuzione delle quattro specie nel territorio in oggetto, cfr. cartinaareale in Casale et al., 2019). Arricchiscono le cenosi cripticole Sphodropsis ghilianii (che, come in buona parte delle valli dell'arco alpino occidentale, si conferma come una della specie ipogeofile più frequenti nelle grotte e in sede microclasica) e Laemostenus obtusus (specie meno comune della precedente, il cui areale copre le Alpi Cozie meridionali, le Marittime, le Liguri e le Langhe). Come elemento più strettamente endogeo (sensu Giachino \& Vailati, 2010) degli orizzonti forestali della valle è inoltre noto Scotodipnus glaber.

Carabidocenosi della fascia subalpina. Come è noto (cfr. Focarile, 1987), la fascia arbustiva rappresenta di norma la zona ecotonale occupata da una carabidofauna molto eterogenea costituita da elementi a diverso praeferendum altitudinale che qui entrano in contatto. Infatti, accanto a elementi delle formazioni forestali trasgressivi verso le quote superiori si osservano specie della fascia alpina che fanno registrare depressioni altimetriche più $\mathrm{o}$ meno marcate. Nel territorio in oggetto, allo stato attuale delle conoscenze, le carabidocenosi che popolano questo orizzonte risultano composte soprattutto da un contingente numeroso di taxa silvicoli del piano montano che sconfinano nelle fasce vegetazionali extrasilvicole, spingendosi talora nel piano alpino (Aptinus alpinus, Carabus solieri, Cychrus italicus, Pterostichus truncatus, P. impressicollis, P. funestes, P. bicolor, Trichotichnus nitens, T. laevicollis e Platynus peirolerii). Gli unici elementi orofili dei quali è stata rilevata la presenza nella fascia arbustiva sono Oreonebria macrodera (osservata nell'Alnetum viridis nei pressi dell'alpeggio di Sella Brignola in Val Corsaglia) e Pterostichus morio (abbondante nei dintorni dello stesso alpeggio e lungo le pendici del Monte Berlino in Val Casotto).

Carabidocenosi degli orizzonti alpino e alto-alpino. La composizione delle carabidocenosi orofile che popolano le testate delle valli ricalca quella osservata più volte in diverse altre valli delle Alpi Liguri. I numerosi reperti ottenuti dagli scriventi in occasioni diverse nei pressi dei Laghi della Brignola (Fig. 6) e, in parte, lungo le pendici del Monte Mondolè ne sono un campione ben rappresentativo. Gli elementi perinivali in senso stretto (caratterizzanti il Nebrietum nivale sensu

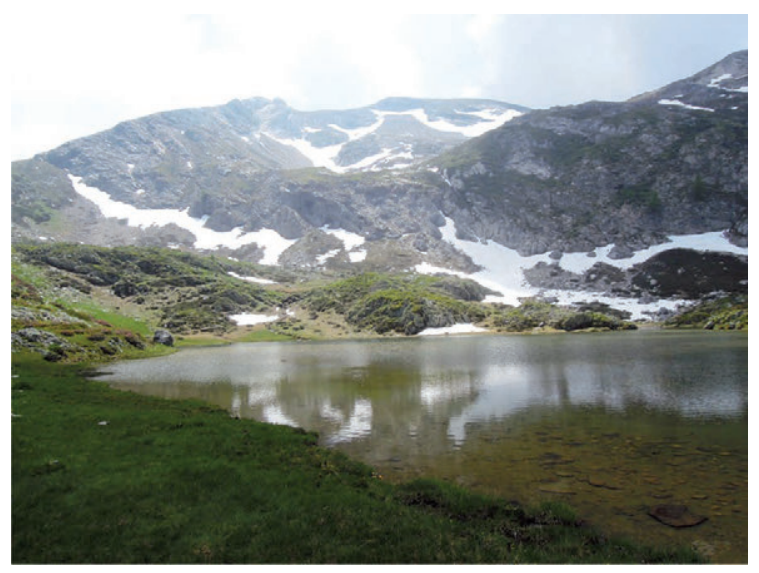

Fig. 6. Il versante Nord della Cima della Brignola e il lago omonimo (foto Luigi Bisio 13.VI.2017). I pendii a monte del lago ospitano una carabidofauna orofila formata da $\mathrm{Ci}$ cindela gallica, Carabus pedemontanus, Oreonebria ligurica, O. macrodera, $O$. angusticollis, Bembidion bipunctatum, B. jacqueti, Stomis elegans, Pterostichus impressicollis, P. morio, P. bicolor, Amara erratica, Harpalus solitaris, Platynus peirolerii e Cymindis humeralis. 
Focarile, 1973) noti sono Oreonebria angusticollis, $O$. ligurica e $O$. macrodera. Oreonebria angusticollis, come di norma per questa specie (cfr. Bisio, 2007), è stata osservata esclusivamente localizzata in paleofrane a grandi blocchi come unica entità ivi presente. Oreonebria ligurica e O. macrodera, che sono invece maggiormente legate a suoli più evoluti già colonizzati dalle fitocenosi erbacee pioniere (la seconda si rinviene spesso anche nell'Alnetum viridis) presentano una distribuzione più continua. In sintopia con queste due ultime specie si osserva spesso il corteggio degli altri abitatori degli ambienti perinivali, attratti dall'elevato tasso di umidità: Carabus pedemontanus (taxon che rispetto alla maggior parte delle specie del subg. Orinocarabus più settentrionali ha una fenologia maggiormente legata al primo fondere delle nevi), C. depressus (la cui fenologia è comunque molto più breve della specie precedente), Cychrus angulicollis (entità caratterizzata da una spiccata criofilìa e da una fenologia alquanto precoce e contratta), Platynus peirolerii, Amara erratica e i Bembidion perinivali caratterizzanti il Testedioletum sensu Focarile, 1973 (Bembidion bipunctatum, B. jacqueti, B. magellense).

Tra le specie che compaiono numerose a inizio estate è anche da annoverare Pterostichus morio, taxon molto comune e diffuso che si osserva in attività per buona parte dell'estate. Con la scomparsa dei nevai, sui terreni che progressivamente sono sempre più interessati da una maggiore xericità superficiale compare il contingente di entità a fenologia più tardiva (Amareto-Cyminditetum sensu Focarile, 1973). Tra le specie censite nel territorio in oggetto sono da annoverare Cymindis humeralis, Amara equestris, A. cardui e Harpalus solitaris.

\section{Carabidocenosi ripicole della Val Corsaglia e della} Val Casotto. Il reticolo idrografico della Val Corsaglia e della Val Casotto ospita una carabidofauna ripicola piuttosto ricca di specie. A grandi linee si possono individuare le seguenti situazioni ambientali:

1. Biotopi sorgivi e torrentelli convoglianti acque di fusione. In buona parte delle Alpi Occidentali, i piccoli torrentelli che in alta quota convogliano acque sorgive e/o di fusione sono la sede elettiva delle specie di Nebria del gruppo "laticollis", entità frigofile stenoterme la cui presenza è condizionata dalla bassa temperatura delle acque. Nelle Alpi Liguri questo gruppo è rappresentato da Nebria (Nebriola) morula K.
Daniel \& J. Daniel, 1891 taxon a tutt'oggi sconosciuto di questo territorio. La sua presenza non si può tuttavia escludere, essendo essa nota della Val Ellero (cfr. Bisio et al., 2015b). La mancanza di dati per il territorio in oggetto è probabilmente da imputare a difetto di ricerche specializzate che tengano conto delle peculiari abitudini di vita di queste due entità che vivono spesso sommerse sotto muschi (cfr. Binaghi, 1951). Altre specie più comuni che popolano questi torrentelli sono Bembidion geniculatum e B. incognitum.

2. I Torrenti Corsaglia e Casotto nella parte alta delle due valli. Nella parte alta della Valli Corsaglia e Casotto gli alvei dei Torrenti Corsaglia e Casotto sono piuttosto incassati (per diversi tratti quasi inaccessibili) e sono caratterizzati da greti discontinui, costituiti da detriti mediamente grossolani (soprattutto pietrame), caratteristica granulometrica che permane a grandi linee rispettivamente sino agli abitati di Corsaglia e di Pamparato valle e che influenza in modo marcato la composizione della carabidofauna ripicola. Il nucleo di Bembidiini già citati si arricchisce: oltre al già citato Bembidion geniculatum, compaiono $B$. varicolor, $B$. ascendens, B. tibiale, B. monticola, B. decorum, $B$. tetracolum e Sinechostictus ruficornis. Per quanto concerne gli altri Carabidi, sono state osservate popolazioni di Nebria rufescens e $N$. jockischii nei pressi del Castello di Casotto.

3. I Torrenti Corsaglia e Casotto nella parte bassa delle due valli. A valle delle due località citate la riduzione delle pendenze consente la sedimentazione di detriti meno grossolani (soprattutto pietrame, ma, a tratti, anche ghiaia e sabbia), situazione che permane sino alla confluenza dei due torrenti e che influenza in modo marcato la composizione della carabidofauna ripicola differenziandola in parte ed arricchendola con un maggior numero di specie. Nebria jockischii e N. rufescens sono sostituite da N. psammodes (molto comune) e da $N$. picicornis (più rara e localizzata), mentre agli onnipresenti Bembidion varicolor, $B$. ascendens, $B$. geniculatum, $B$. tibiale, $B$. monticola, $B$. decorum, $B$. tetracolum e Sinechostictus ruficornis si aggiungono Bembidion quadrimaculatum, $B$. articulatum, B. punctulatum, B. genei, Sinechostictus 
decoratus e, molto più rari, $S$. doderoi e $S$. inustus. Sono inoltre da segnalare tra gli elementi ripicoli censiti nella bassa valle Chlaeniellus vestitus, Chlaenius velutinus, Abacetus salzmanni, Agonum viduum, Limodromus assimilis e $\mathrm{Pa}$ ranchus albipes.

\section{ConClusioni}

Le 159 specie di Carabidae note delle valli in oggetto rappresentano il 54\% delle 294 che, secondo un elenco elaborato a partire dalla lista fornita da $\mathrm{Ca}-$ sale \& Vigna Taglianti (1993) e aggiornato successivamente con nuovi dati tratti dalla letteratura e con reperti inediti, sono note per le Alpi Liguri. La distribuzione percentuale dei corotipi (Tab. 2 e Fig. 7) evidenzia per il territorio in oggetto un tasso di endemicità elevato $(22 \%$ del totale delle entità note, conseguenza della presenza di ben 36 taxa ad areale ristretto). Tra gli elementi endemici prevalgono gli alpini sud-occidentali (18, pari a oltre $1 ' 11 \%$ del totale delle specie) e, come già osservato in altre valli delle Alpi Liguri (cfr. Bisio et al., 2013, 2015b; Bisio \& Giuntelli, 2019), sono numerosi anche gli alpino-appenninici ( 8 , pari al $5 \%$ ). Il $79 \%$ delle specie censite è formato invece dalla compagine di elementi a più ampia distribuzione della quale fanno parte un'elevata quota $(45 \%)$ di entità a gravitazione più settentrionale (oloartici sensu lato), una frazione minore, ma ancora rilevante, di taxa a corotipo europeo $(32 \%)$ e un gruppo numericamente trascurabile di specie mediterranee $(2 \%)$.
Tab. 2. Distribuzione percentuale dei corotipi.

\begin{tabular}{llcc}
\hline & Corotipi & Numero & $\%$ \\
\hline Oloartici & & & \\
& OLA & 9 & 5,66 \\
& PAL & 12 & 7,55 \\
& WPA & 1 & 0,63 \\
& ASE & 19 & 11,95 \\
SIE & 17 & 10,69 \\
CEM & 1 & 0,63 \\
& CAE & 2 & 1,26 \\
TEM & 4 & 2,52 \\
& TUE & 5 & 3,14 \\
AFP & 1 & 0,63 \\
\hline
\end{tabular}

\begin{tabular}{llcc}
\hline Europei & & & \\
& EUM & 6 & 3,77 \\
& EUR & 21 & 13,21 \\
& CEU & 16 & 10,06 \\
& SEU & 6 & 3,77 \\
\hline Mediterranei & & & \\
& MED & 2 & 1,26 \\
& WME & 1 & 0,63 \\
\hline \multirow{6}{*}{ Endemici } & & & \\
& ALSW & 18 & 11,32 \\
& ALPW & 7 & 4,40 \\
& ALPS & 3 & 1,89 \\
& ALAP & 8 & 5,03 \\
\hline
\end{tabular}

Totale

100,00

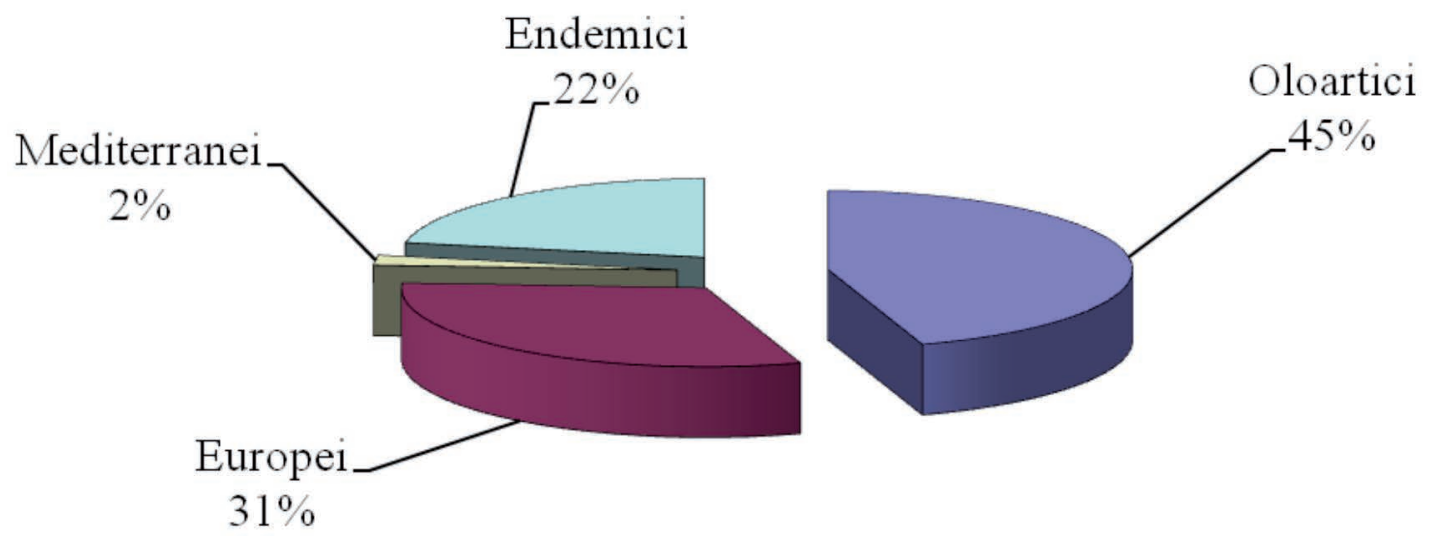

Fig. 7. Distribuzione percentuale dei corotipi nelle principali categorie corologiche. 


\section{RINGRAZIAMENTI}

Desideriamo ringraziare gli amici Achille Casale e Gianni Allegro per la lettura critica del testo, per i preziosi suggerimenti e per la comunicazione di alcuni dati corologici, così come Matteo Negro che ha redatto la tavola relativa alla valle in oggetto.

\section{BIBLIOGRAFIA}

Allegro G., 2014 - Carabidi della Langa Astigiana (Piemonte meridionale) (Coleoptera, Carabidae). Rivista Piemontese di Storia Naturale, 35: 151-165.

Allegro G., Bertolino S., Mazza A., Molinari R., 2004 - La carabidofauna della Riserva Naturale Speciale del Torrente Orba (Alessandria, Piemonte) (Coleoptera, Carabidae). Rivista Piemontese di Storia Naturale, 25: 257-275.

Allegro G., Cersosimo M., 2004 - I Carabidi del Parco naturale regionale di Rocchetta Tanaro (Asti, Piemonte) (Coleoptera Carabidae). Bollettino del Museo regionale di Scienze naturali di Torino, 21: 123-144.

Allegro G., Cersosimo M., Palestrini C., 2002 - I Carabidi dell'Oasi WWF 'Bosco del Lago' di Castello di Annone (Asti, Piemonte) (Coleoptera, Carabidae). Rivista Piemontese di Storia Naturale, 23: 175-194.

BAUdi Di Selve F., 1871 - Sulle specie italiane di Scotodipnus. Bullettino della Società Entomologica Italiana, 3: 25-35.

Baudi Di Selve F., 1890 - Catalogo dei Coleotteri del Piemonte. Annali della Regia Accademia dell'Agricoltura Torino, 32: 51-274.

BenAzZI M., Gourbault N., 1977 - Atrioplanaria morisii n. sp., a new cave planarian from Italy. Bollettino di Zoologia, 44: 327-335

BinAGHI G., 1935 - Note su alcuni Carabidi. Memorie della Società Entomologica Italiana, 14: 63-64.

BinaGHI G., 1936 - Studio critico del Sottogenere Scotodipnus s. str. (Col. Carabidae). Bollettino della Società Entomologica Italiana, 68: 80-91.

BinAGHI G., 1939 - Lo Sphodropsis Ghilianii Schaum, le sue razze e la sua diffusione nelle Alpi occidentali (Col. Carabidae). Memorie della Società Entomologica Italiana, 18: 177-185.

BinAGHI G., 1951 - Coleotteri d'Italia. Vita, ambienti, utilità, danni, mezzi di lotta. Briano, Genova, 210 pp.

Bisio L., 1995 - Contributo alla conoscenza di alcuni Pterostichus orofili del Piemonte (Coleoptera Carabidae). Rivista Piemontese di Storia Naturale, 15: 67-98.

Bisio L., 2000 - Secondo contributo alla conoscenza di Carabus del subg. Orinocarabus del Piemonte: Carabus putzeysianus e il gruppo fairmairei-cenisius-heteromorphus (Coleoptera Carabidae). Rivista Piemontese di Storia Naturale, 21: 115152.

Bisio L., 2001 - Note sulla carabidofauna della Valle Angrogna (Val Pellice, Alpi Cozie) (Coleoptera Carabidae). Rivista Piemontese di Storia Naturale, 22: 183-223.

Bisio L., 2007 - Contributo alla conoscenza di Oreonebria del Piemonte e della Valle d'Aosta: Oreonebria (Oreonebria) angusticollis (Bonelli, 1809) (Coleoptera Carabidae). Rivista Piemontese di Storia Naturale, 28: 165-180.

Bisıo L., 2008 - Secondo contributo alla conoscenza di Oreonebria del Piemonte e della Valle d'Aosta: Oreonebria castanea e le specie del gruppo "picea" (Coleoptera Carabidae). Rivista Piemontese di Storia Naturale, 29: 177-209.

Bisio L., 2009a - Note corologiche e ecologiche su alcuni Bembidiini del Piemonte e della Valle d'Aosta (Coleoptera Carabidae). Rivista Piemontese di Storia Naturale, 30: 57-77.

BISIO L., 2009b - Note corologiche e ecologiche su alcuni Bembidiini perinivali delle Alpi Occidentali italiane (Coleoptera Carabidae). Rivista Piemontese di Storia Naturale, 30: 95-129.

Bisio L., 2009c - Terzo contributo alla conoscenza del gen. Amara Bonelli, 1810 in Piemonte e in Valle d'Aosta: osservazioni sulle specie del subg. Percosia (Coleoptera Carabidae). Rivista Piemontese di Storia Naturale, 30: 149-161.

Bisıo L., 2015 - I Coleotteri Carabidi della Valpelline (Valle d'Aosta) (Coleoptera Carabidae). Bollettino della Società Entomologica Italiana, 147: 51-74.

Bisio L., 2019 - Note corologiche ed ecologiche su Bembidion (Peryphanes) italicum De Monte, 1943 e su Bembidion (Peryphanes) pallidicorne G. Müller, 1921 in Piemonte e in Valle d'Aosta (Coleoptera Carabidae). Rivista Piemontese di Storia Naturale, 40: 239-253.

Bisio L., Allegro G., Giuntelli P., 2016 - I Coleotteri Carabidi della Valle di Cogne (Valle d'Aosta) (Coleoptera Carabidae). Revue Valdôtaine d'Histoire Naturelle, 70: 75-112. 
Bisio L., Allegro G., Giuntelli P., 2017 - Note corologiche ed ecologiche su alcuni Leistus del Piemonte e della Valle d'Aosta: una sintesi (Coleoptera Carabidae). Rivista Piemontese di Storia Naturale, 38: 225-273.

Bisio L., Giachino P.M., Allegro G., 2015a - I Coleotteri Carabidi della Valle Orco (Alpi Graie) (Coleoptera Carabidae). Rivista Piemontese di Storia Naturale, 36: 89-144.

Bisio L., Giachino P.M., Allegro G., Giuntelli P., 2015b - I Coleotteri Carabidi della Val Ellero e della Val Maudagna (Alpi Liguri) (Coleoptera Carabidae). Rivista Piemontese di Storia Naturale, 36: 171-214.

Bisio L., Giuntelli P., 2006 - Note sulla carabidofauna delle Valli di Lanzo (Alpi Graie) (Coleoptera Carabidae). Rivista Piemontese di Storia Naturale, 27: 221-267.

Bisio L., Giuntelli P., 2011 - I Coleotteri Carabidi della Val Maira (Coleoptera Carabidae). Rivista Piemontese di Storia Naturale, 32: 173-226.

Bisio L., Giuntelli P., 2014 - I Coleotteri Carabidi della Val Grana (Alpi Cozie) (Coleoptera Carabidae). Rivista Piemontese di Storia naturale, 35: 167-212.

Bisio L., Giuntelli P., 2019 - I Coleotteri Carabidi della Val Tanaro (Alpi Liguri) (Coleoptera Carabidae). Rivista Piemontese di Storia Naturale, 40: 177-229.

Bisio L., Giuntelli P., Allegro G., 2013 - I Coleotteri Carabidi della Val Pesio (Alpi Liguri) (Coleoptera Carabidae). Memorie della Società Entomologica Italiana, 90: 61-87.

Bisio L., Giuntelli P., Allegro G., 2018 - I Coleotteri Carabidi della Val Vermenagna (Alpi Marittime e Liguri) (Coleoptera Carabidae). Rivista Piemontese di Storia Naturale, 39: 223-265.

Bisio L., Negro M., Giuntelli P., 2015c - I Coleotteri Carabidi della Valtournenche (Valle d'Aosta) (Coleoptera Carabidae). Revue Valdôtaine d'Histoire Naturelle, 69: 99-136.

Bologna M.A., Vigna Taglianti A., 1982 - Il popolamento cavernicolo delle Alpi Occidentali. Lavori della Società Italiana di Biogeografia, 7: 515-544.

Bologna M.A., Vigna Taglianti A., 1985 - Fauna cavernicola delle Alpi Liguri. Annali del Museo Civico di Storia Naturale G. Doria, Genova, 84 bis: 1-399.

BonaVita P., Vigna TAgLianti A., 2005 - Le Alpi orientali come zona di transizione nel popolamento dei bembidini (Coleoptera Carabidae). Biogeographia (Biogeografia delle Alpi e prealpi centro-orientali), 26: 203-228.

Bordoni A., 1968 - Le stazioni liguri più orientali di Sphodropsis ghilianii ghilianii Sch. ed Actenipus obtusus Chd. s.l. (Coleoptera). Bollettino dell'Associazione Romana di Entomologia, 23: 51-52.

Breuning S., 1932-1936 - Monographie der Gattung Carabus L. Bestimmungs-Tabelle europäischen Coleopteren, 104-110, Reitter, Troppau, 1610 pp. 41 tavv.

CAPRA F., 1941 - Note su alcuni Pterostichus italiani (Coleoptera Carabidae). Bollettino della Società Entomologica Italiana, 72: $147-156$.

Casale A., 1977 - Reperti di Licinus (s. str.) depressus (Paykull) in Valle d'Aosta. Sistematica del genere Licinus e delle specie italiane del "gruppo depressus" (Col. Carabidae). Revue Valdôtaine d'Histoire Naturelle, 31: 84-92.

Casale A., 1988 - Revisione degli Sphodrina (Coleoptera, Carabidae, Sphodrini). Monografie del Museo Regionale di Scienze Naturali di Torino, 5: $1024 \mathrm{pp}$.

CAsale A., Cavazzuti P.F., 1975 - Sul Carabus (Chrysocarabus) solieri Dejean in Italia (Col. Carabidae): sistematica, corologia, ecologia. Bollettino dell'Istituto di Entomologia della Università di Bologna, 32: 231-255.

Casale A., CAvazzuti P.F., 1976 - Sistematica e corologia di Carabus (Orinocarabus) putzeysianus Géhin, con descrizione di una nuova sottospecie, e nota sinonimica (Coleoptera Carabidae). Fragmenta Entomologica, 12: 285-297.

Casale A., Giachino P.M., 2010 - Due nuovi Coleotteri ipogei delle Alpi Occidentali: Duvalius (Duvalius) lanai n. sp. (Carabidae: Trechini) e Archeoboldoria sturanii n. sp. (Cholevidae: Leptodirinae). Rivista Piemontese di Storia Naturale, 31 : 213-240.

Casale A., Giachino P.M., Lana E., 2019 - Note sul genere Duvalius nelle Alpi occidentali e nel'Appennino Ligure, con descrizione di due nuovi taxa ipogei del Piemonte: Duvalius (Duvalius) chestai n. sp. e Duvalius (Duvalius) gestroi cristianae n. ssp. (Coleoptera, Carabidae: Trechini). Rivista Piemontese di Storia Naturale, 40: 317-353.

Casale A., Sturani M., Vigna Taglianti A., 1982 - Coleoptera Carabidae I. Introduzione, Paussinae, Carabinae. Fauna d'Italia, XVIII, Calderini, Bologna, 499 pp.

Casale A., Vigna Taglianti A., Brandmayr P., Colombetta G., 2006 - Insecta Coleoptera Carabidae (Carabini, Cychrini, 
Trechini, Abacetini, Stomini, Pterostichini). In: Ruffo S., Stoch F. (eds.), Ckmap (Checklist and distribution of the Italian fauna). Memorie del Museo Storia Naturale Verona, 2. serie, Sezione Scienze della vita, 17: 159-164, with data on CDRom.

Casale A., Vigna Taglianti A., 1993 - I Coleotteri Carabidi delle Alpi occidentali e centro-occidentali (Coleoptera Carabidae). Biogeographia (Il popolamento delle Alpi occidentali), 16: 331-399.

Dal Piaz G.V. (coord.), 1991 - Guide Geologiche Regionali, vol. 2, Alpi Liguri. Pubblicazione a cura della Società Geologica Italiana. Ed. Be.Ma., 293 pp.

Daniel K., Daniel J., 1898 - Das Amaren-Subgenus Leirides Putz. Coleopteren Studien. München, 2: 31-34.

Daniel K., 1906 - Beiträge zur Koleopteren-Geographie. Münchener Koleopterologischen Zeitschrift, 3: 41-45.

Degiovanni A., Magrini P., 2016 - Note tassonomiche su alcuni Trechus italiani del "gruppo subnotatus" (sensu Jeannel, 1927), con rivalutazione di un taxon e una nuova sinonimia (Coleoptera, Carabidae, Trechinae). Giornale italiano di entomologia, 14: 375-394

Della Beffa G., 1911 - I Coleotteri dell'agro torinese loro rapporti con la vegetazione e l'agricoltura. Annali della Regia Accademia di Agricoltura di Torino, 54: 69-346.

Dellepiane G., 1924 - Guida per escursioni nelle Alpi e Appennini Liguri. Bollettino C.A.I., sez. Ligure, Genova, XXIII + 494 pp.

Deuve T., 2004 - Illustrated catalogue of the genus Carabus of the world (Coleoptera: Carabidae). Pensoft, Sofia-Moscow, $462 \mathrm{pp}$.

FoCARILE A., 1973 - Sulla Coleotterofauna alticola del Gran San Bernardo (versante valdostano). Annali della Facoltà di Agraria dell'Università di Torino, 9: 51-118.

FoCARILE A., 1987 - Ecologie et Biogeographie des Coléoptères de haute altitude en Vallée d'Aoste. Regione Autonoma Valle d'Aosta. Assessorato Agricoltura, Foreste e Ambiente naturale, 167 pp., 72 carte, 4 tavv.

GANGLbauer L., 1900 - Revision der europäisch-mediterranen Arten der blinden Bembidiinen-Genera. Verhandlungen des Zoologisch-Botanischen Vereins in Wien, 50: 151-184.

Ghiliani V., 1887 - Elenco delle specie di Coleotteri trovate in Piemonte (opera postuma a cura di L. Camerano). Annali Regia Accademia Agricoltura Torino, 29: 195-381.

Giachino P.M., 1993 - La distribuzione dei generi Binaghites e Bathysciola nelle Alpi Occidentali (Coleoptera Carabidae e Cholevidae). Biogeographia, Lavori della Società Italiana di Biogeografia, (n.s.) 16: 401-404.

Giachino P.M., Casale A., 1983 - Sistematica e morfologia genitale di alcuni Pterostichus italiani (Coleoptera Carabidae). Bollettino del Museo Regionale di Scienze naturali, 1: 417-450.

Giachino P.M., Vailati D., 2010 - The subterranean environment. Hypogean life, concepts and collecting techniques. WBA Handbooks, 3, Verona: 132 pp.

Giachino P.M., VAILATi D., 2016 - Riflessioni sulla terminologia biospeleologica: i concetti di troglobio, troglofilo e troglosseno. Atti del Convegno Nazionale "La ricerca carsologica in Italia", 22-23 giugno 2013, Laboratorio carsologico sotterraneo di Bossea, Frabosa Soprana (pubbl. 1 giugno 2016): 195-200.

Gregoretti F., 1991 - Interesse naturalistico e scientifico della Grotta di Bossea. Atti dell'incontro di Bossea 14-15 settembre 1991, Laboratorio carsologico sotterraneo di Bossea, Frabosa Soprana, 23-41.

HIEKE F., 1978 - Revision der Amara-Untergattung Percosia Zimm. und Bemerkungen zu anderen Amara-Arten (Coleoptera Carabidae). Deutsche Entomologische Zeitschrift 25: 215-326.

JAEGER B., 2008 - Die westpaläarktischen Arten der Bradycellus-Untergattung Bradycellus Erichson 1837 unter besonderer Berücksichtigung des Mittelmeerraumes (Coleoptera, Carabidae). Linzer biologische Beiträge, 40 (2): 1509-1577.

JEANNEl R., 1942 - Coléoptères Carabiques II (Faune de France, vol. 40). P. Lechevalier (Paris), 573-1173.

Lana E., Casale A., Giachino P.M., 2014 - Attività biospeleologica 2012-2013. “Grotte”, Bollettino del Gruppo Speleologico Piemontese, GSP CAI-UGET, Torino, 56: 41-64.

Lana E., Casale A., Giachino P.M., Grafitti G., 2008 - Attività biospeleologica 2006-2007. “Grotte”, Bollettino del Gruppo Speleologico Piemontese, GSP CAI-UGET, Torino, 50: 40-46.

Lana E., Casale A., Giachino P.M., Chesta M., Balestra V., 2018 - Attività biospeleologica 2017. "Grotte”, Bollettino del Gruppo Speleologico Piemontese, GSP CAIUGET, Torino, 60: 38-47

Luigioni P., 1929 - I Coleotteri d'Italia. Catalogo sinonimico-topografico-bibliografico. Memorie della Pontificia Accademia delle Scienze "I nuovi Lincei”, Roma, 13: 1-1160. 
Magistretti M., 1965 - Fauna d'Italia, VIII. Coleoptera: Cicindelidae, Carabidae. Catalogo topografico. Calderini, Bologna, $512 \mathrm{pp}$.

Magrini P., Degiovanni A., 2012 - Note sulla validità specifica di Trechus nicoleae Moncoutier, 1986 (Coleoptera, Carabidae). Fragmenta Entomologica, 44: 13-18.

MARtinotti A., 1968 - Elenco sistematico e geografico della fauna cavernicola del Piemonte e della Valle d'Aosta. Rassegna Speleologica Italiana, 20: 3-34.

MonZINI V., 1986 - Reperti inediti nel Parco del Ticino. Bollettino della Società Entomologica Italiana, 118: 127-134.

Morisi A., 1969 - Il laboratorio sotterraneo di Bossea. Primi risultati. Mondo Ipogeo, Cuneo: 35-38.

Morisi A., 1970 - Rendiconto Biospeleologico per il 1970. Mondo Ipogeo, Cuneo: 56-60.

Morisi A., 1971a - Attività biospeleologica 1970-71. Mondo Ipogeo, Cuneo: 48-51.

Morisi A., 1971b - Un cavernicolo alla volta: Sphodropsis ghilianii (Schaum). Mondo Ipogeo, Cuneo: 57-59, 1 tav.

Morisi A., 1972a - Note faunistiche per l'anno 1971-1972. Mondo Ipogeo, Cuneo: 52-56.

Morisi A., 1972b - Risultati biospeleologici preliminari della campagna estiva alla Tana del Forno 114 Pi, Serra di Pamparato. Mondo Ipogeo, Cuneo: 56-57.

Morisi A., 1973a - Un nuovo Actenipus Jeann. delle Alpi Cozie (Coleoptera Carabidae). Memorie della Società Entomologica Italiana, 80: 65-86.

Morisi A., 1973b - Attività biospeleologica 1973. Mondo Ipogeo, Cuneo, 8: 60-62.

MÜLler G., 1926 - I Coleotteri della Venezia Giulia. I Adephaga. Studi Entomologici, 306 pp.

MÜLLER G., 1930 - I Coleotteri cavernicoli italiani. Elenco geografico delle grotte con indicazione delle specie e varietà dei coleotteri cavernicoli finora trovati in Italia. Le Grotte d'Italia, 4: 65-85.

Neri P., Bonavita P., Gudenzi I., Magrini P., Toledano L., 2011 - Bembidiina della fauna italo-corsa: chiavi di identificazione (Insecta Coleoptera Carabidae). Quaderno di Studi e Notizie di Storia Naturale della Romagna, 33: 1-183.

Neri P., Bonavita P., Vigna Taglianti A., Gudenzi I., 2013 - Note tassonomiche nomenclatoriali ( $3^{\circ}$ contributo) su alcuni Bembidiina della Fauna Italiana (Insecta Coleoptera Carabidae). Quaderno di Studi e Notizie di Storia Naturale della Romagna, 36: 89-95.

Peano G., 1973 - La stazione scientifica di Bossea. Mondo Ipogeo, Cuneo, 8:44-50.

PeAno G., 1974 - Attività della stazione scientifica del G.S.A.M. nella grotta di Bossea. Atti del XI Congresso Nazionale di Speleologia, Genova, 1972, Rassegna Speleologica Italiana, Memorie, 11: 295-297.

PEAno G., 1999 - Il fenomeno carsico nelle Alpi Liguri. In: Smiraglia (coord.): L'originalità naturalistica e culturale delle Alpi Liguri nei loro rapporti con l'Appennino Ligure e con le Alpi Marittime. Atti dell'incontro di Nava-Pornassio (IM) 1415 settembre 1996, 96 pp.

Pesarini C., MonzInI V., 2011 - Insetti della Fauna Italiana. Coleotteri Carabidi I. Società italiana di Scienze naturali, Milano, $152 \mathrm{pp}$.

RAVIZZA C., 1972 - I Bembidion popolanti gli orizzonti montano, subalpino e alpino della Valle d'Aosta. Studi sui Bembidion. IX Contributo (Coleoptera Carabidae). Memorie della Società Entomologica Italiana, 51: 94-122.

Regione Piemonte, 1998 - Precipitazioni e temperature. Banca Dati Climatologica del Piemonte - Archivio Precipitazioni. CDRom.

SCIAKY R., 1984 - Revisione dei Pterostichus italiani affini a cristatus Duf. (XII Contributo alla conoscenza dei Coleoptera Carabidae). Bollettino del Museo di Storia Naturale di Verona, 11: 149-170.

SChatzMAYr A., 1930 - I Pterostichus italiani. Memorie della Società Entomologica Italiana, 8: 145-339.

SChATZMAYR A., 1942 - Appunti Coleotterologici VII. Natura, 33: 58-60.

SChAtZmaYr A., 1943 - Appunti coleotterologici. VIII. Natura, Rivista di Scienze naturali, Milano, 34: 91-97.

STRANEO L., 1933 - Appunti su alcuni Carabidi italiani. Bollettino della Società Entomologica Italiana, 65: 113-115.

STRANeo L., 1935 - Contributo alla conoscenza dei Carabidi degli Abruzzi. Memorie della Società Entomologica Italiana, 14: 59-62.

Vigna Taglianti A., 1966 - Reperti: Carabus glabratus latior Born (Coleoptera Carabidae). Bollettino dell'Associazione Romana di Entomologia, 20: 60.

Vigna Taglianti A., 1982 - Le attuali conoscenze sui Coleotteri Carabidi cavernicoli italiani. Biogeographia, Lavori della Società Italiana di Biogeografia, (n.s.) 7: 339-430. 
Vigna Taglianti A., 2005 - Checklist e corotipi delle specie di Carabidae della fauna italiana. Appendice B, pp. 186-225. In: P. Brandmayr, T. Zetto \& R. Pizzolotto (eds.), I Coleotteri Carabidi per la valutazione ambientale e la conservazione della biodiversità. Manuale operativo: APAT, Manuali e Linee Guida, 34: 240 pp.

Vigna Taglianti A., Casale A., 1973 - Due nuovi Duvalius delle Alpi Liguri e considerazioni sul gruppo del Duvalius carantii (Coleoptera, Carabidae), Fragmenta Entomologica, 9: 109-134.

Vigna Taglianti A., Spettoli R., Brandmayr P., Algieri M.C., 2001 - Note tassonomiche e corologiche su Carabus granulatus in Italia, con descrizione di una nuova sottospecie di Calabria (Coleoptera Carabidae). Memorie della Società Entomologica Italiana, 80: 65-86.

Wolf B., 1937 - Animalium Cavernarum Catalogus. Volumen II. Junk, Wien (1934-1937), 616 pp.

Wolf B., 1938 - Animalium Cavernarum Catalogus. Volumen III: Animalium Catalogus. Junk, Wien (1934-1938), 918 pp.

ZANELla L., 2017 - Taxonomic note on Abax contractus (Heer, 1841) and description of A. parallelepipedus ligurinus n. subsp. (Coleoptera, Carabidae, Pterostichini). Zootaxa, 4238: 366-374. 